\title{
Ramsey numbers of cubes versus cliques
}

\author{
David Conlon * Jacob Fox ${ }^{\dagger} \quad$ Choongbum Lee ${ }^{\ddagger} \quad$ Benny Sudakov ${ }^{\S}$
}

\begin{abstract}
The cube graph $Q_{n}$ is the skeleton of the $n$-dimensional cube. It is an $n$-regular graph on $2^{n}$ vertices. The Ramsey number $r\left(Q_{n}, K_{s}\right)$ is the minimum $N$ such that every graph of order $N$ contains the cube graph $Q_{n}$ or an independent set of order $s$. In 1983, Burr and Erdös asked whether the simple lower bound $r\left(Q_{n}, K_{s}\right) \geq(s-1)\left(2^{n}-1\right)+1$ is tight for $s$ fixed and $n$ sufficiently large. We make progress on this problem, obtaining the first upper bound which is within a constant factor of the lower bound.
\end{abstract}

\section{Introduction}

For graphs $G$ and $H$, the Ramsey number $r(G, H)$ is defined to be the smallest natural number $N$ such that every red/blue edge-coloring of the complete graph $K_{N}$ on $N$ vertices contains a red copy of $G$ or a blue copy of $H$.

One obvious construction, noted by Chvátal and Harary [16], which gives a lower bound for these numbers is to take $\chi(H)-1$ disjoint red cliques of size $|G|-1$ and to connect every pair of vertices which are in different cliques by a blue edge. If $G$ is connected, the resulting graph contains neither a red copy of $G$ nor a blue copy of $H$, so that $r(G, H) \geq(|G|-1)(\chi(H)-1)+1$. Burr [8] strengthened this bound by noting that if $\sigma(H)$ is the smallest color class in any $\chi(H)$-coloring of the vertices of $H$, we may add a further red clique of size $\sigma(H)-1$, obtaining

$$
r(G, H) \geq(|G|-1)(\chi(H)-1)+\sigma(H) .
$$

Following Burr and Erdős [8, 11], we say that a graph $G$ is $H$-good if the Ramsey number $r(G, H)$ is equal to this bound. If $\mathcal{G}$ is a family of graphs, we say that $\mathcal{G}$ is $H$-good if all sufficiently large graphs in $\mathcal{G}$ are $H$-good. When $H=K_{s}$, where $\sigma\left(K_{s}\right)=1$, we simply say that $G$ or $\mathcal{G}$ is $s$-good.

The classical result on Ramsey goodness, which predates the definition, is the theorem of Chvátal [15] showing that all trees are $s$-good for any $s$. On the other hand, the family of trees is not $H$-good for every graph $H$. For example [13], a construction of $K_{2,2}$-free graphs due to Brown [7] allows one to show that there is a constant $c<\frac{1}{2}$ such that

$$
r\left(K_{1, t}, K_{2,2}\right) \geq t+\sqrt{t}-t^{c}
$$

*Mathematical Institute, Oxford OX1 3LB, United Kingdom. Email: david.conlon@maths.ox.ac.uk. Research supported by a Royal Society University Research Fellowship.

${ }^{\dagger}$ Department of Mathematics, MIT, Cambridge, MA 02139-4307. Email: fox@math.mit.edu. Research supported by a Packard Fellowship, a Simons Fellowship, an MIT NEC Corp. award and NSF grant DMS-1069197.

${ }^{\ddagger}$ Department of Mathematics, MIT, Cambridge, MA 02139-4307. Email: cb_lee@mit.edu. Research supported in part by a Samsung Scholarship.

${ }^{\S}$ Department of Mathematics, ETH, 8092 Zurich, Switzerland and Department of Mathematics, UCLA, Los Angeles, CA 90095. Email: benjamin.sudakov@math.ethz.ch. Research supported in part by SNSF grant 200021-149111 and by a USA-Israel BSF grant. 
for $t$ sufficiently large. This is clearly larger than $\left(\left|K_{1, t}\right|-1\right)\left(\chi\left(K_{2,2}\right)-1\right)+\sigma\left(K_{2,2}\right)=t+2$.

In an effort to determine what properties contribute to being Ramsey good, Burr and Erdös [9, 11 ] conjectured that if $\Delta$ is fixed then the family of graphs with bounded maximum degree $\Delta$ is $s$-good for any $s$ (and perhaps even $H$-good for all $H$ ). This conjecture holds for bipartite graphs $H$ [12] but is false in general, as shown by Brandt [6]. He proved that for $\Delta \geq \Delta_{0}$ almost every $\Delta$-regular graph on a sufficiently large number of vertices is not even 3-good. His result (and a similar result in [30]) actually proves something stronger, namely, that if a graph $G$ has strong expansion properties then it cannot be 3 -good.

On the other hand, it has been shown that if a family of graphs exhibits poor expansion properties then it will tend to be good [1, 30]. To state the relevant results, we define the bandwidth of a graph $G$ to be the smallest number $\ell$ for which there exists an ordering $v_{1}, \ldots, v_{n}$ of the vertices of $G$ such that every edge $v_{i} v_{j}$ satisfies $|i-j| \leq \ell$. This parameter is known to be intimately linked to the expansion properties of the graph. In particular, any bounded-degree graph with poor expansion properties will have sublinear bandwidth [4].

The first such result, shown by Burr and Erdös [11], states that for any fixed $\ell$ the family of connected graphs with bandwidth at most $\ell$ is $s$-good for any $s$. This result was recently extended by Allen, Brightwell and Skokan [1, who showed that the set of connected graphs with bandwidth at most $\ell$ is $H$-good for every $H$. Their result even allows the bandwidth $\ell$ to grow at a reasonable rate with the size of the graph $G$. If $G$ is known to have bounded maximum degree, their results are particularly strong, saying that for any $\Delta$ and any fixed graph $H$ there exists a constant $c$ such that if $G$ is a graph on $n$ vertices with maximum degree $\Delta$ and bandwidth at most $c n$ then $G$ is $H$-good.

Many of the original problems of Burr and Erdös [11] have now been resolved [30] but one that remains open is to determine whether the family of hypercubes is $s$-good for every $s$. The hypercube $Q_{n}$ is the graph on vertex set $\{0,1\}^{n}$ where two vertices are connected by an edge if and only if they differ in exactly one coordinate. This family of graphs has sublinear bandwidth but does not have bounded degree, so the result of Allen, Brightwell and Skokan does not apply.

To get a first bound for $r\left(Q_{n}, K_{3}\right)$, note that a simple greedy embedding implies that any graph with maximum degree $d$ and at least $d n+2^{n}$ vertices has a copy of $Q_{n}$ in its complement. Suppose now that the edges of a complete graph have been 2-colored in red and blue and there is neither a blue triangle nor a red copy of $Q_{n}$. Then, since the blue neighborhood of any vertex forms a red clique, the maximum degree in blue is at most $2^{n}-1$. Hence, the graph must have at most $\left(2^{n}-1\right) n+2^{n}<2^{n}(n+1)$ vertices. We may therefore conclude that $r\left(Q_{n}, K_{3}\right) \leq 2^{n}(n+1)$.

It is not hard to extend this argument to show that for any $s$ there exists a constant $c_{s}$ such that $r\left(Q_{n}, K_{s}\right) \leq c_{s} 2^{n} n^{s-2}$. This is essentially the best known bound. Here we improve this bound, obtaining the first upper bound which is within a constant factor of the lower bound.

Theorem 1.1. For any natural number $s \geq 3$, there exists a constant $c_{s}$ such that

$$
r\left(Q_{n}, K_{s}\right) \leq c_{s} 2^{n}
$$

The original question of Burr and Erdös [1] relates to $s$-goodness but it is natural to also ask whether the family of cubes is $H$-good for any $H$. For bipartite $H$, this follows directly from a result of Burr, Erdős, Faudree, Rousseau and Schelp [12]. Our result clearly implies that for any $H$, there is a constant $c_{H}$ such that $r\left(Q_{n}, H\right) \leq c_{H} 2^{n}$.

For triangles, the rough idea of the proof is to show that if a red/blue edge-coloring of $K_{N}$ does 
not contain a blue triangle then it may be tiled with a collection of red cliques which have low blue density between them. A red copy of $Q_{n}$ may then be found by inserting subcubes into each of the red cliques and patching them together using the fact that the blue density between these different cliques is low. Throughout the argument, it is very important to keep close control on the size and number of the red cliques as the definition of low blue density in a particular bipartite subgraph will depend on the size of the two cliques forming its endpoints.

For $K_{4}$, the method is an extension of this idea, except the tiling will now consist of cliques which are free of blue triangles, again with low blue density between different cliques. In order to make this useful for embedding cubes, we then have to perform a second level of tiling, splitting each such clique into red subcliques with low density between them, as was already done for triangles. In general, when we consider $K_{s}$, there will be $s-2$ levels of tiling to keep track of. This makes the bookkeeping somewhat complex. Accordingly, we have chosen to present the proof in stages, first considering triangles, then $K_{4}$ and only then the general case. Although this leads to some redundancies, it allows us to introduce the additional concepts needed for each step at a reasonable pace.

More generally, one may ask which families of graphs are $s$-good for all $s$. A powerful result proved by Nikiforov and Rousseau [30] shows that graphs with small separators are $s$-good. They used this result to resolve a number of the original questions of Burr and Erdős [1] regarding Ramsey goodness. Let the degeneracy $d(G)$ of a graph $G$ be the smallest natural number $d$ such that every induced subgraph of $G$ has a vertex of degree at most $d$. Furthermore, we say that a graph $G$ has a $(t, \eta)$-separator if there exists a vertex subset $T \subseteq V(G)$ such that $|T| \leq t$ and every connected component of $V(G) \backslash T$ has size at most $\eta|V(G)|$. The result of Nikiforov and Rousseau then says that for any $s \geq 3, d \geq 1$ and $0<\gamma<1$, there exists $\eta>0$ such that the class $\mathcal{G}$ of $d$-degenerate graphs $G$ with a $\left(|V(G)|^{1-\gamma}, \eta\right)$-separator is $s$-good.

We will apply this theorem, together with the Alon-Seymour-Thomas separator theorem for graphs with a forbidden minor [2], to show that for any $s \geq 3$ any family of graphs with a forbidden minor is $s$-good. A graph $H$ is said to be a minor of $G$ if $H$ can be obtained from a subgraph of $G$ by contracting edges. By an $H$-minor of $G$, we mean a minor of $G$ which is isomorphic to $H$. For a graph $H$, let $\mathcal{G}_{H}$ be the family of graphs which do not contain an $H$-minor.

Theorem 1.2. For every fixed graph $H$, the class $\mathcal{G}_{H}$ of graphs $G$ which do not contain an $H$-minor is $s$-good for all $s \geq 3$.

In particular, since the family of planar graphs consists exactly of those graphs which do not contain $K_{5}$ or $K_{3,3}$ as a minor, we have the following corollary.

Corollary 1.1. The family of planar graphs is $s$-good for all $s \geq 3$.

A minor-closed family $\mathcal{G}$ is a collection of graphs which is closed under taking minors. The graph minor theorem of Robertson and Seymour [31] states that any minor-closed family of graphs may be characterized by a finite collection of forbidden minors. We say that a minor-closed family is nontrivial if it is not the class consisting of all graphs. Note that the following corollary is an immediate consequence of Theorem 1.2 as any nontrivial minor-closed family $\mathcal{G}$ is a subfamily of $\mathcal{G}_{H}$, where $H$ is a graph not in $\mathcal{G}$.

Corollary 1.2. Any nontrivial minor-closed family of graphs $\mathcal{G}$ is $s$-good for all $s \geq 3$. 
We will begin, in Section 2, by studying the Ramsey number of cubes versus triangles. We will then show, in Section 3, how our arguments extend to $K_{4}$ before treating the general case in Section 4. In Section 5, we will prove Theorem 1.2. We will conclude with some further remarks. All logarithms are base 2 unless otherwise indicated. For the sake of clarity of presentation, we systematically omit floor and ceiling signs whenever they are not crucial. We also do not make any serious attempt to optimize absolute constants in our statements and proofs.

\section{Triangle versus cube}

The argument works for $n \geq 6$. Consider a coloring of the edges of the complete graph $K_{N}$ on the vertex set $[N]=\{1,2, \ldots, N\}$ for $N \geq 7000 \cdot 2^{n}$ with two colors, red and blue, and assume that there are no blue triangles. We will prove that this coloring contains a red $Q_{n}$.

\subsection{Preprocessing the coloring}

For each $d=0,1,2, \ldots, \log n+3$, we use the following procedure to construct a family $\mathcal{S}$ of subsets of $[N]$ (note that $\log n+3 \leq n$ for $n \geq 6$ ):

If there exists a set $S$ which induces a red clique of order exactly $4 \cdot 2^{n-d}$, then arbitrarily choose one, add it to the family $\mathcal{S}$, and remove the vertices of the clique from $[N]$. We define the codimension $d(S)$ of such a set as $d(S)=d$. When there are no more such red cliques, continue to the next value of $d$. In the end, if we have $\sum_{S \in \mathcal{S}}|S| \geq \frac{N}{2}$, then we let $\mathcal{S}$ be our family. Otherwise, if $\sum_{S \in \mathcal{S}}|S|<\frac{N}{2}$, we add the set of remaining vertices to $\mathcal{S}$, and declare it to have codimension zero (note that this set has size at least $\frac{N}{2} \geq 4 \cdot 2^{n}$ ).

In either of the cases, we have $\sum_{S \in \mathcal{S}}|S| \geq \frac{N}{2}$. Note that we also have the following properties.

Proposition 2.1. (i) For an integer $i \geq 1$, let $X=\bigcup_{S \in \mathcal{S}, d(S) \geq i} S$. Then each vertex $v \in[N]$ has at most $2^{n-i+3}$ blue neighbors in $X$.

(ii) For every set $S \in \mathcal{S}$, the subgraph induced by $S$ has maximum blue degree at most $\frac{2^{n-d(S)}}{2 n}$.

Proof. (i) Given $i \geq 1$ and a set $X$ defined as above, note that no subset of $X$ induces a red clique of size at least $4 \cdot 2^{n-i+1}=2^{n-i+3}$, since such a set would have been added to $\mathcal{S}$ in the previous round. On the other hand, since there are no blue triangles, the blue neighborhood of every vertex induces a red clique. Therefore, for every $v \in[N], v$ has at most $2^{n-i+3}$ blue neighbors in $X$.

(ii) The claim is trivially true for $d>0$, since every set of codimension at least 1 is a red clique. For $d=0$, if $S$ is not a red clique, then it is the set of codimension zero added to $\mathcal{S}$ after the sets of codimensions 0 to $\log n+3$. Thus, there are no subsets of $S$ which induce a red clique of size at least $4 \cdot 2^{n-(\log n+3)}$, since such a set would have been added earlier to the family $\mathcal{S}$. On the other hand, since there are no blue triangles, the blue neighborhood of every vertex induces a red clique. Therefore, every vertex of $S$ has at most $4 \cdot 2^{n-(\log n+3)} \leq \frac{2^{n}}{2 n}$ blue neighbors in $S$.

\subsection{Tiling the cube}

Our strategy is to first decompose the cube $Q_{n}$ into smaller cubes and then to embed it piece by piece into $K_{N}$, placing one of the subcubes in each of the subsets from $\mathcal{S}$. We represent the subcubes 
of $Q_{n}$ by using vectors in $\{0,1, *\}^{n}$. For example, the vector $(0,1, *, \ldots, *)$ will represent the subcube $\left\{\left(0,1, x_{1}, \ldots, x_{n-2}\right):\left(x_{1}, \ldots, x_{n-2}\right) \in\{0,1\}^{n-2}\right\}$.

We seek a tiling of $Q_{n}$, which we define as a collection of vertex-disjoint cubes that covers all the vertices of $Q_{n}$ and uses only cubes that have all their fixed coordinates at the start of the vector. That is, they are of the form $C=\left(a_{1}, a_{2}, \ldots, a_{d}, *, *, \ldots, *\right)$ for some $d \geq 0$ and $a_{1}, a_{2}, \ldots, a_{d} \in\{0,1\}$. We will call such a cube special and let $d$ be the codimension $d(C)$ of $C$. We say that two disjoint cubes $C$ and $C^{\prime}$ are adjacent, if there are vertices $x \in C$ and $x^{\prime} \in C^{\prime}$ which are adjacent in $Q_{n}$. Note that the following list of properties holds for such cubes and a tiling composed from them.

Proposition 2.2. (i) If two special cubes $C$ and $C^{\prime}$ intersect, then we have $C \subset C^{\prime}$ or $C^{\prime} \subset C$.

(ii) Two disjoint cubes $C=\left(a_{1}, \ldots, a_{d}, *, \ldots, *\right)$ and $C^{\prime}=\left(b_{1}, \ldots, b_{d^{\prime}}, *, \ldots, *\right)$ for $d^{\prime} \leq d$ are adjacent if and only if $\left(a_{1}, \ldots, a_{d^{\prime}}\right)$ and $\left(b_{1}, \ldots, b_{d^{\prime}}\right)$ differ in exactly one coordinate.

(iii) For a tiling $\mathcal{C}$ and a special cube $C \in \mathcal{C}$ of codimension $d$, there are at most $d$ other special cubes $C^{\prime} \in \mathcal{C}$ of codimension $d\left(C^{\prime}\right) \leq d$ which are adjacent to $C$.

Proof. (i) Let $d=d(C), d^{\prime}=d\left(C^{\prime}\right)$ and, without loss of generality, suppose that $d^{\prime} \leq d$. Suppose that $\left(a_{1}, \ldots, a_{n}\right) \in C \cap C^{\prime}$. Then, since we are only considering special cubes, we must have $C=$ $\left(a_{1}, \ldots, a_{d}, *, \ldots, *\right)$ and $C^{\prime}=\left(a_{1}, \ldots, a_{d^{\prime}}, *, \ldots, *\right)$. However, we then have $C \subset C^{\prime}$.

(ii) If $\left(a_{1}, \ldots, a_{d^{\prime}}\right)=\left(b_{1}, \ldots, b_{d^{\prime}}\right)$, then we have $C \subset C^{\prime}$, and thus we may assume that this is not the case. Cubes $C$ and $C^{\prime}$ are adjacent if and only if there are two vectors $\mathbf{v}=\left(a_{1}, \ldots, a_{d}, x_{d+1}, \ldots, x_{n}\right)$ and $\mathbf{w}=\left(b_{1}, \ldots, b_{d^{\prime}}, y_{d^{\prime}+1}, \ldots, y_{n}\right)$ in $\{0,1\}^{n}$ which differ in exactly one coordinate. However, since the two vectors restricted to the first $d^{\prime}$ coordinates are already different, this can happen only if $\left(a_{1}, \ldots, a_{d^{\prime}}\right)$ and $\left(b_{1}, \ldots, b_{d^{\prime}}\right)$ differ in exactly one coordinate. Moreover, one can see that if this is the case, then there is an assignment of values to $x_{i}$ and $y_{j}$ so that $\mathbf{v}$ and $\mathbf{w}$ indeed differ in exactly one coordinate.

(iii) Since $C$ has codimension $d$, there are only $d$ coordinates that one can 'flip' from $C$ to obtain a cube adjacent to $C$.

As we have already mentioned, our tiling $\mathcal{C}$ of the cube $Q_{n}$ will be constructed in correspondence with the family $\mathcal{S}$ constructed in Section 2.1. We will construct the tiling $\mathcal{C}$ by finding cubes of the tiling one by one. We slightly abuse notation and use $\mathcal{C}$ also to denote the 'partial' tiling, where only part of the cube $Q_{n}$ is covered. At each step, we will find a subcube $C$ which covers some non-covered part of $Q_{n}$ and assign it to some set $S_{C} \in \mathcal{S}$. We say that such an assignment is proper if the following properties hold.

\section{Proper assignment.}

1. If $C$ has codimension $d$, then $S_{C}$ has codimension $d$.

2. Suppose that $C$ is adjacent to some cube $C^{\prime}$ already in the tiling and that $C^{\prime}$ is assigned to $S_{C^{\prime}}$. Then the bipartite graph induced by $S_{C}$ and $S_{C^{\prime}}$ contains at most $\frac{\left|S_{C}\right|\left|S_{C^{\prime}}\right|}{16 \delta^{2}}$ blue edges, where $\delta=\max \left\{d(C), d\left(C^{\prime}\right)\right\}$.

Our algorithm for finding the tiling $\mathcal{C}$ and the corresponding sets in $\mathcal{S}$ is as follows.

Tiling Algorithm. At each step, consider all possible special cubes $C$ which 
(a) are disjoint from the cubes $C^{\prime} \in \mathcal{C}$,

(b) have $d(C) \geq d\left(C^{\prime}\right)$ for all $C^{\prime} \in \mathcal{C}$, and

(c) for which there exists a set $S \in \mathcal{S}$ which has not yet been assigned and such that assigning $C$ to $S$ is a proper assignment.

Take a cube $C_{0}$ of minimum codimension satisfying (a), (b) and (c) and add it to the tiling. Assign $C_{0}$ to the set $S_{C_{0}} \in \mathcal{S}$ given by (c).

The following proposition shows that the algorithm will terminate successfully.

Proposition 2.3. If the tiling is not complete, then the algorithm always chooses a cube from a non-empty collection.

Proof. If $\mathcal{S}$ contains a set of codimension zero, then the algorithm will choose the whole cube $Q_{n}$ in the first step and assign it to a set of codimension zero. In this case we have a trivial tiling and there is nothing to prove.

Thus, we may assume that $\mathcal{S}$ contains no set of codimension zero. If this is the case, note that for all $C \in \mathcal{C}$, we have $\left|S_{C}\right|=4|C|$. Let $\mathcal{S}^{\prime \prime}$ be the subfamily of $\mathcal{S}$ consisting of sets which are already assigned to some cube in $\mathcal{C}$ and let $\mathcal{S}^{\prime}=\mathcal{S} \backslash \mathcal{S}^{\prime \prime}$. Note that

$$
\left|\bigcup_{S \in \mathcal{S}^{\prime}} S\right|=\left|\bigcup_{S \in \mathcal{S}} S\right|-\left|\bigcup_{S \in \mathcal{S}^{\prime \prime}} S\right| \geq \frac{N}{2}-\left|\bigcup_{C \in \mathcal{C}} S_{C}\right| \geq 3500 \cdot 2^{n}-4 \cdot 2^{n} \geq 3496 \cdot 2^{n} .
$$

For each $i$, let $\mathcal{S}_{i}^{\prime}=\left\{S \in \mathcal{S}^{\prime}: d(S)=i\right\}$. If $\left|\mathcal{S}_{i}^{\prime}\right| \leq 32 i^{3}$ for all $i$, then we have

$$
\left|\bigcup_{S \in \mathcal{S}^{\prime}} S\right|=\sum_{i}\left|\bigcup_{S \in \mathcal{S}_{i}^{\prime}} S\right| \leq \sum_{i} 32 i^{3} \cdot 4 \cdot 2^{n-i}=128 \cdot 2^{n} \sum_{i} \frac{i^{3}}{2^{i}}=3328 \cdot 2^{n},
$$

which is a contradiction (we used the fact that $\sum_{i} \frac{i^{3}}{2^{i}}=26$ ). Therefore, there exists an index $i$ for which $\left|\mathcal{S}_{i}^{\prime}\right|>32 i^{3}$. We have $i>0$ since $\mathcal{S}$ contains no set of codimension zero.

Since the tiling is not complete, there exists a vertex $\left(a_{1}, \ldots, a_{n}\right) \in Q_{n}$ which is not yet covered by any of the cubes already in $\mathcal{C}$. Consider the cube $C=\left(a_{1}, \ldots, a_{i}, *, \ldots, *\right)$. By Proposition 2.2, there are at most $i$ cubes in our partial tiling $\mathcal{C}$ of codimension at most $i$ which are adjacent to this cube. Let $C_{1}, \ldots, C_{j}$ for $j \leq i$ be these cubes and fix an index $a \leq j$. We say that a set $S \in \mathcal{S}_{i}^{\prime}$ is bad for $S_{C_{a}}$ if there are at least $\frac{1}{16 i^{2}}\left|S_{C_{a}} \| S\right|$ blue edges between $S_{C_{a}}$ and $S$. We claim that there are at most $32 i^{2}$ sets in $\mathcal{S}_{i}^{\prime}$ which are bad for $S_{C_{a}}$.

Let $X_{i}=\bigcup_{S \in \mathcal{S}_{i}^{\prime}} S$ and note, by Proposition 2.1(i), that there are at most $\left|S_{C_{a}}\right| \cdot 2^{n-i+3}$ blue edges between the sets $S_{C_{a}}$ and $X_{i}$. Each set $S \in \mathcal{S}_{i}^{\prime}$ which is bad for $S_{C_{a}}$ accounts for at least $\frac{1}{16 i^{2}}\left|S_{C_{a}}\right||S|=$ $\frac{2^{n-i+3}}{32 i^{2}}\left|S_{C_{a}}\right|$ such blue edges (note that $|S|=4 \cdot 2^{n-i}$ ). Therefore, in total, there are at most $32 i^{2}$ sets in $\mathcal{S}_{i}^{\prime}$ which are in bad relation with $S_{C_{a}}$, as claimed above. Since $\left|\mathcal{S}_{i}^{\prime}\right|>32 i^{3}$, there exists a set $S \in \mathcal{S}_{i}^{\prime}$ which is not bad for any of the sets $S_{C_{a}}$ for $1 \leq a \leq j \leq i$.

Suppose that in the previous step we embedded some cube of codimension $d$. In order to show that $C$ satisfies (a) and (b), it suffices to verify that $i \geq d$, since $i \geq d$ implies the fact that $C$ is disjoint from all the other cubes in $\mathcal{C}$ (if $C$ intersects some other cube, then that cube must contain $C$ and therefore also contains $\left(a_{1}, \ldots, a_{n}\right)$ by Proposition 2.2). Furthermore, if this is the case, assigning $C$ 
to $S$ is a proper assignment (thus we have (c)), since $C_{1}, \ldots, C_{j}$ are the only cubes adjacent to $C$ already in the tiling.

Suppose, for the sake of contradiction, that $i<d$ and consider the time $t$ immediately after we last embedded a cube of codimension at most $i$. At time $t$, since $\left(a_{1}, \ldots, a_{n}\right)$ was not covered, the cubes in $\mathcal{C}$ are disjoint from $C$. Moreover, the set of cubes of codimension at most $i$ which are adjacent to $C$ is the same as at the current time. Therefore, $C$ could have been added to the tiling at time $t$ as well, contradicting the fact that we always choose a cube of minimum codimension. Thus we have $i \geq d$, as claimed.

Note that as an outcome of our algorithm, we obtain a tiling $\mathcal{C}$ for which every pair of adjacent cubes $C, C^{\prime} \in \mathcal{C}$ have at most $\frac{1}{16 \delta^{2}}\left|S_{C}\right|\left|S_{C^{\prime}}\right|$ blue edges between $S_{C}$ and $S_{C^{\prime}}$, where $\delta=\max \left\{d(C), d\left(C^{\prime}\right)\right\}$.

\subsection{Imposing a maximum degree condition}

Having constructed the tiling $\mathcal{C}$ and made the assignment of the cubes to sets in $\mathcal{S}$, we now wish to impose certain maximum degree conditions between the sets $S_{C}$ for $C \in \mathcal{C}$. For a set $C \in \mathcal{C}$ of codimension $d=d(C)$, let $C_{1}, \ldots, C_{j}$ be the cubes of codimension at most $d$ which are adjacent to $C$. Note that we have $j \leq d$ by Proposition 2.2. Moreover, there are at most $\frac{1}{16 d^{2}}\left|S_{C}\right|\left|S_{C_{a}}\right|$ blue edges between $S_{C}$ and $S_{C_{a}}$ for all $a \leq j$.

Now, for each $a$, remove all the vertices in $S_{C}$ which have at least $\frac{1}{8 d}\left|S_{C_{a}}\right|$ blue neighbors in $S_{C_{a}}$ and let $T_{C}$ be the subset of $S_{C}$ left after these removals. Since there are at most $\frac{1}{16 d^{2}}\left|S_{C}\right|\left|S_{C_{a}}\right|$ blue edges between $S_{C}$ and $S_{C_{a}}$, for each index $a$, we remove at most $\frac{\left|S_{C}\right|}{2 d}$ vertices from $S_{C}$, and thus the resulting set $T_{C}$ is of size at least $\frac{\left|S_{C}\right|}{2} \geq 2 \cdot 2^{n-d}$. Note that all the vertices in $T_{C}$ have blue degree at most $\frac{1}{8 d}\left|S_{C_{a}}\right| \leq \frac{1}{4 d}\left|T_{C_{a}}\right|$ in the set $T_{C_{a}}$. That is, we have the following property.

Maximum degree condition. For each pair of adjacent cubes $C, C^{\prime} \in \mathcal{C}$ with $d(C) \geq$ $d\left(C^{\prime}\right)$, every vertex in $T_{C}$ has at most $\frac{1}{4 d(C)}\left|T_{C^{\prime}}\right|$ blue neighbors in the set $T_{C^{\prime}}$.

\subsection{Embedding the cube}

We now show how to embed $Q_{n}$. Recall that $Q_{n}$ was tiled by cubes in $\mathcal{C}$, each corresponding to a subset from the family $\mathcal{S}$. We will greedily embed these cubes one by one into their assigned sets from the family $\mathcal{S}$, in decreasing order of their codimensions. If there are several cubes of the same codimension, then we arbitrary choose the order between them.

For each $C \in \mathcal{C}$, we will greedily embed the vertices of $C$ into the set $T_{C} \subseteq S_{C}$. Let $d=d(C)$. Suppose that we are about to embed $x \in C$ and let $f: Q_{n} \rightarrow[N]$ denote the partial embedding of the cube $Q_{n}$ obtained so far. Note that $x$ has at most $d$ neighbors $x_{1}, x_{2}, \ldots, x_{j}$ (for $j \leq d$ ) which are already embedded and belong to a cube other than $C$. Since we have only embedded cubes of codimension at least $d$, the maximum degree condition imposed in Section 2.3 implies that all the vertices $f\left(x_{i}\right)$ have blue degree at most $\frac{1}{4 d}\left|T_{C}\right|$ in the set $T_{C}$. Together, these neighbors forbid at most $\frac{1}{4}\left|T_{C}\right|$ vertices of $T_{C}$ from being the image of $x$.

In addition, $x$ has at most $n-d$ neighbors $y_{1}, \ldots, y_{k}$ (for $k \leq n-d$ ) which are already embedded and belong to $C$. By Proposition 2.1, each vertex $f\left(y_{i}\right)$ has blue degree at most $\frac{2^{n-d}}{2 n}$ in the set $T_{C}$. Together, these neighbors forbid at most $\frac{2^{n-d}}{2}$ vertices of $T_{C}$ from being the image of $x$. Finally, 
there are at most $2^{n-d}-1$ vertices in $T_{C}$ which are images of some other vertex of $C$ that is already embedded. Therefore, the number of vertices in $T_{C}$ into which we cannot embed $x$ is at most

$$
\frac{1}{4}\left|T_{C}\right|+\frac{1}{2} \cdot 2^{n-d}+\left(2^{n-d}-1\right)
$$

which is less than $\left|T_{C}\right|$ since $\left|T_{C}\right| \geq 2 \cdot 2^{n-d}$. Hence, there exists a vertex in $T_{C}$ which we can choose as an image of $x$ to extend the current partial embedding of the cube. Repeating this procedure until we embed the whole cube $Q_{n}$ completes the proof.

\section{Cliques of order 4}

The argument for general cliques is similar to that for triangles given in the previous section. However, there are several new concepts involved. To slowly develop the necessary concepts, we first provide a proof of the next case, which is $K_{4}$ versus a cube.

Recall that in the triangle case, we started by iteratively finding sets which formed a red clique (see Section 2.11). Red cliques were a natural choice, since the blue neighborhood of every vertex formed a red clique. Either we were able to find large red cliques or we were able to restrict the maximum blue degree of vertices in some way (see Proposition 2.1). If one attempts to employ the same strategy for the $K_{4}$ case, then the natural choice of sets that we should take instead of red cliques are blue triangle-free sets, since the blue neighborhood of every vertex now forms a blue triangle-free set. Suppose that we have found a family $\mathcal{S}_{1}$ of blue triangle-free sets. Since blue $K_{3}$-free sets are not as powerful as red cliques for embedding subgraphs, we repeat the whole argument within each blue triangle-free set $S \in \mathcal{S}_{1}$, to obtain red cliques which are subsets of $S$. By so doing, we obtain a second family of sets $\mathcal{S}_{2}$, consisting of red cliques. We refer to sets in $\mathcal{S}_{\ell}$ as level $\ell$ sets and, for a set $S \in \mathcal{S}_{\ell}$, we define its level as $\ell(S)=\ell$.

In order to find an embedding of the cube $Q_{n}$ using a strategy similar to that used in the triangle case, we wish to find a tiling of $Q_{n}$ and, for each cube $C$ in the tiling, a red clique $S_{C} \in \mathcal{S}_{2}$ so that for two adjacent cubes $C$ and $C^{\prime}$, the sets $S_{C}$ and $S_{C^{\prime}}$ stand in 'good' relation. However, directly finding such a tiling and an assignment is somewhat difficult since we do not have good control on the blue edges between the sets in $\mathcal{S}_{2}$. To be more precise, suppose that we are given $S_{1}, S_{1}^{\prime} \in \mathcal{S}_{1}$ and subsets $S_{2} \subset S_{1}, S_{2}^{\prime} \subset S_{1}^{\prime}$ in $\mathcal{S}_{2}$. Then the control on the blue edges between $S_{2}$ and $S_{2}^{\prime}$ is 'inherited' from the control on the blue edges between $S_{1}$ and $S_{1}^{\prime}$, and thus depends on the relative sizes of $S_{1}$ and $S_{1}^{\prime}$, not on the relative sizes of $S_{2}$ and $S_{2}^{\prime}$ as in the triangle case (unless $S_{1}=S_{1}^{\prime}$ ). To circumvent this difficulty, we will need to maintain tight control on the edge density between different sets in $\mathcal{S}_{1}$ as well as those in $\mathcal{S}_{2}$.

We seek a double tiling, which is defined to be a pair $\mathcal{C}=\mathcal{C}_{1} \cup \mathcal{C}_{2}$ of tilings satisfying the property that for every $C_{2} \in \mathcal{C}_{2}$ there exists a cube $C_{1} \in \mathcal{C}_{1}$ such that $C_{2} \subset C_{1}$ (in other words, $\mathcal{C}_{2}$ is a refined tiling of $\mathcal{C}_{1}$ ). We refer to cubes in $\mathcal{C}_{\ell}$ as level $\ell$ cubes and, for a cube $C \in \mathcal{C}_{\ell}$, we define its level as $\ell(C)=\ell$. Our goal is to find, for each $\ell=1,2$, an assignment of cubes in $\mathcal{C}_{\ell}$ to sets in $\mathcal{S}_{\ell}$. The following are the key new concepts involved in the $K_{4}$ case.

Definition 3.1. $\quad$ (i) For a cube $C \in \mathcal{C}_{1}$, we define its 1-codimension as $d_{1}(C)=d(C)$. For a cube $C \in \mathcal{C}_{2}$ contained in a cube $C_{1} \in \mathcal{C}_{1}$, we define its 1-codimension as $d_{1}(C)=d\left(C_{1}\right)$ and its 2-codimension as $d_{2}(C)=d(C)-d\left(C_{1}\right)$. 
(ii) Suppose $C, C^{\prime} \in \mathcal{C}$ are adjacent. We say that they have level 1 adjacency if the level 1 cubes containing $C$ and $C^{\prime}$ are different. Otherwise, they have level 2 adjacency (note that $C$ and $C^{\prime}$ are both level 2 cubes in the second case).

After finding the double tiling, in order to find an embedding of the cube, we only consider the level 2 tiling (as explained above, we need to go through a level 1 tiling in order to obtain a 'nice' level 2 tiling). For two adjacent cubes $C, C^{\prime} \in \mathcal{C}_{2}$, the definition of the corresponding sets $S_{C}, S_{C^{\prime}}$ being in 'good relation' (see the definition of proper assignment in Section 3.2) now depends on the type of adjacency they have. If they have level 1 adjacency, then the good relation will be defined in terms of their 1-codimensions and if they have level 2 adjacency, then it will be in terms of their 2-codimensions. By doing this, we can overcome the above mentioned difficulty of not having enough control on the blue edges between $S_{C}$ and $S_{C^{\prime}}$. Afterwards, we proceed as in the previous section, by imposing a maximum degree condition between cubes in $\mathcal{C}_{2}$ and then embedding the cube.

We now provide the details of the proof. Our argument works for $n \geq 32$. Consider a coloring of the edges of the complete graph $K_{N}$ on the vertex set $[N]$ for $N \geq 2^{46} \cdot 2^{n}$ with two colors, red and blue, and assume that there is no blue $K_{4}$. We prove that this coloring contains a red $Q_{n}$.

\subsection{Preprocessing the coloring}

Note that we have $n \geq 2 \log n+21$ for $n \geq 32$. We use the following procedure to construct our first level $\mathcal{S}_{1}$ of subsets of $[N]$ :

For each $d=0,1,2, \ldots, \log n+18$, if there exists a set $S$ which induces a blue triangle-free graph of order exactly $2^{18} \cdot 2^{n-d}$, then arbitrarily choose one, add it to the family $\mathcal{S}_{1}$, and remove the vertices of $S$ from $[N]$. We define the 1-codimension $d_{1}(S)$ of such a set as $d_{1}(S)=d$. When there are no more such sets, continue to the next value of $d$. If, after running through all values of $d, \sum_{S \in \mathcal{S}}|S|<\frac{N}{2}$, then add the set of remaining vertices to $\mathcal{S}$ and declare it to be an exceptional set with 1-codimension zero (note that this set has size at least $\frac{N}{2} \geq 2^{18} \cdot 2^{n}$ ).

Now we perform a similar decomposition for each set in $\mathcal{S}_{1}$ to construct our second level $\mathcal{S}_{2}$. For each set $S_{1} \in \mathcal{S}_{1}$, consider the following procedure (suppose that $S_{1}$ has codimension $d_{1}=d\left(S_{1}\right)$ ):

1. If $S_{1}$ is not exceptional: for each $d=0,1,2, \ldots, \log n+3$, if there exists a subset $S \subset S_{1}$ which induces a red clique of order exactly $8 \cdot 2^{n-d_{1}-d}$, then arbitrarily choose one, add it to the family $\mathcal{S}_{2}$, and remove the vertices of the clique from $S_{1}$. We define the 2-codimension of such a set as $d_{2}(S)=d$ and the 1-codimension as $d_{1}(S)=d_{1}$. When there are no more such red cliques, continue to the next value of $d$. If, after running through all values of $d, \sum_{S \in \mathcal{S}_{2}, S \subset S_{1}}|S|<\frac{\left|S_{1}\right|}{2}$, then add the set of remaining vertices to $\mathcal{S}_{2}$, and declare it to be an exceptional set with 2-codimension zero and 1-codimension $d_{1}$ (note that this set has size at least $\frac{\left|S_{1}\right|}{2} \geq 8 \cdot 2^{n-d_{1}}$ ).

2. If $S_{1}$ is exceptional: add $S_{1}$ to the family $\mathcal{S}_{2}$. Define the 2-codimension of $S_{1} \in \mathcal{S}_{2}$ as zero and its 1-codimension as zero.

Let $\mathcal{S}=\mathcal{S}_{1} \cup \mathcal{S}_{2}$ (we suppose that $\mathcal{S}$ is a multi-family and, thus, if a set is in both $\mathcal{S}_{1}$ and $\mathcal{S}_{2}$, then we have two copies of this set in $\mathcal{S}$, however they can be distinguished by their levels). For a set $S \in \mathcal{S}$, define its codimension as $d(S)=\sum_{i \leq \ell(S)} d_{i}(S)$. 
Proposition 3.1. (i) $\sum_{S \in \mathcal{S}_{1}}|S| \geq \frac{N}{2}$ and, for every $S_{1} \in \mathcal{S}_{1}$, we have $\sum_{S \in \mathcal{S}_{2}, S \subset S_{1}}|S| \geq \frac{\left|S_{1}\right|}{2}$.

(ii) For an integer $i \geq 1$, let $X=\bigcup_{S \in \mathcal{S}_{1}, d_{1}(S) \geq i} S$. Then each vertex $v \in[N]$ has at most $2^{19} \cdot 2^{n-i}$ blue neighbors in $X$.

(iii) For a set $S_{1} \in \mathcal{S}_{1}$ and an integer $i \geq 1$, let

$$
X=\bigcup_{S \in \mathcal{S}_{2}, S \subset S_{1}, d_{2}(S) \geq i} S .
$$

Then each vertex $v \in S_{1}$ has at most $16 \cdot 2^{n-d_{1}\left(S_{1}\right)-i}$ blue neighbors in $X$.

(iv) For every set $S \in \mathcal{S}_{2}$, the subgraph induced by $S$ has maximum blue degree at most $\frac{2^{n-d(S)}}{n}$.

Proof. Part (i) is clear and we omit the proof of (ii), since its proof is similar to that of part (i) of Proposition 2.1.

(iii) Suppose that $S_{1}$ is an exceptional set. Then $S_{1}$ is the unique set $S$ satisfying $S \in \mathcal{S}_{2}$ and $S \subset S_{1}$. Since $d_{2}\left(S_{1}\right)=0, X$ is an empty set for every $i \geq 1$. Thus the conclusion follows.

Now suppose that $S_{1}$ is not an exceptional set. Since $S_{1}$ induces a blue triangle-free set, a blue neighborhood in $S_{1}$ of a vertex $v \in S_{1}$ forms a red clique. Thus, if a vertex $v \in S_{1}$ has more than $16 \cdot 2^{n-d_{1}\left(S_{1}\right)-i}$ blue neighbors in $X$, then inside the neighborhood of $v$ we can find a set inducing a red clique of size at least $8 \cdot 2^{n-d_{1}\left(S_{1}\right)-(i-1)}$. However, this set must have been added in the previous step. Therefore, there are no such vertices.

(iv) Suppose that $S \subset S_{1}$ with $S_{1} \in \mathcal{S}_{1}$. If both $S$ and $S_{1}$ are not exceptional sets, then $S$ induces a red clique and the conclusion follows. Suppose now that $S$ is exceptional and $S_{1}$ is not. Then, as in (iii), we can see that there is no vertex in $S$ which has at least $8 \cdot 2^{n-d_{1}(S)-(\log n+3)}=\frac{2^{n-d(S)}}{n}$ blue neighbors in $S$. One can similarly handle the case when $S_{1}$ is exceptional, since we have $S=S_{1}$ in this case.

\subsection{Tiling the cube}

The following proposition is similar to Proposition 2.2 (we omit its proof).

Proposition 3.2. Let $\mathcal{C}=\mathcal{C}_{1} \cup \mathcal{C}_{2}$ be a double tiling, and let $C \in \mathcal{C}$ be a special cube of codimension $d$.

(i) If $C$ is a level 1 cube, then, for each $\ell=1,2$, there are at most $d_{1}(C)$ special cubes of level $\ell$ and codimension at most $d$ which are adjacent to $C$ (and $C$ has level 1 adjacency with all these cubes).

(ii) If $C$ is a level 2 cube, then there are at most $d_{2}(C)$ special cubes of codimension at most $d$ which have level 2 adjacency with $C$ (they are necessarily of level 2) and, for $\ell=1,2$, at most $d_{1}(C)$ cubes of level $\ell$ and codimension at most $d$ which have level 1 adjacency with $C$.

Our double tiling $\mathcal{C}$ of the cube $Q_{n}$ will be constructed in correspondence with the family $\mathcal{S}$ constructed in Section 3.1. We will construct $\mathcal{C}$ by finding cubes of the tiling one by one. We slightly abuse notation and use $\mathcal{C}$ also to denote the 'partial' double tiling, where only part of the cube $Q_{n}$ is covered. Ideally, we would like to construct $\mathcal{C}$ by constructing the level 1 tiling $\mathcal{C}_{1}$ first and then the level 2 tiling $\mathcal{C}_{2}$. However, as we will soon see, it turns out that constructing the tiling in increasing order of codimension is more effective than in increasing order of level. At each step, we find a 
subcube $C$ which covers some non-covered part of $Q_{n}$ and assign it to some set $S_{C} \in \mathcal{S}$. We say that such an assignment is proper if the following properties hold.

\section{Proper assignment.}

1. $\ell(C)=\ell\left(S_{C}\right)$ and, for $\ell=\ell(C)$, we have $d_{\ell}(C)=d_{\ell}\left(S_{C}\right)$.

2. If $C_{2} \subset C_{1}$ with $C_{1} \in \mathcal{C}_{1}$ and $C_{2} \in \mathcal{C}_{2}$, then $S_{C_{2}} \subset S_{C_{1}}$.

3. Suppose that $C$ is adjacent to some cube $C^{\prime}$ which is already in the tiling, where $C^{\prime}$ is assigned to $S_{C^{\prime}}$ and $C$ and $C^{\prime}$ have level $\rho$ adjacency. Then the number of blue edges in the bipartite graph induced by $S_{C}$ and $S_{C^{\prime}}$ is at most $\left|S_{C}\right|\left|S_{C^{\prime}}\right| \cdot(8 \delta)^{\ell(C)+\ell\left(C^{\prime}\right)-6}$, where $\delta=\max \left\{d_{\rho}(C), d_{\rho}\left(C^{\prime}\right)\right\}$.

Our algorithm for finding the tiling $\mathcal{C}$ and the corresponding sets in $\mathcal{S}$ is as follows.

Tiling Algorithm. At each step, consider all possible special cubes $C$ which

(a) can be added to $\mathcal{C}$ to extend the partial tiling,

(b) have $d(C) \geq d\left(C^{\prime}\right)$ for all $C^{\prime} \in \mathcal{C}$, and

(c) for which there exists a set $S \in \mathcal{S}$ which has not yet been assigned and such that assigning $C$ to $S$ gives a proper assignment.

Take a cube $C_{0}$ of minimum codimension satisfying (a), (b) and (c) and add it to the tiling. Assign $C_{0}$ to the set $S_{C_{0}} \in \mathcal{S}$ given by (c).

Condition (a) is equivalent to saying that either $C$ is disjoint from all cubes in $\mathcal{C}_{1}$ or is contained in some cube in $\mathcal{C}_{1}$ and is disjoint from all cubes in $\mathcal{C}_{2}$. The following proposition shows that the algorithm will terminate successfully.

Proposition 3.3. If the tiling is not complete, then the algorithm always chooses a cube from a non-empty collection.

Proof. Suppose that in the previous step we embedded some cube of codimension $d$ (let $d=0$ for the first iteration of the algorithm). Since the tiling is not complete, there exists a vertex $\left(a_{1}, \ldots, a_{n}\right) \in Q_{n}$ which is not covered twice.

Case 1: $\left(a_{1}, \ldots, a_{n}\right)$ is not covered by any of the cubes in $\mathcal{C}_{1}$.

Let $\mathcal{S}^{\prime \prime}$ be the subfamily of $\mathcal{S}_{1}$ consisting of sets which are already assigned to some cube in $\mathcal{C}_{1}$, and let $\mathcal{S}^{\prime}=\mathcal{S}_{1} \backslash \mathcal{S}^{\prime \prime}$. If $\mathcal{S}^{\prime \prime}$ contains a set of codimension zero, then the corresponding cube is the whole cube $Q_{n}$, contradicting the fact that $\left(a_{1}, \ldots, a_{n}\right)$ is not covered. Thus we may assume that there is no set of codimension zero in $\mathcal{S}^{\prime \prime}$, from which it follows that $\left|S_{C}\right|=2^{18}|C|$ for all $C \in \mathcal{C}_{1}$. Note that

$$
\left|\bigcup_{S \in \mathcal{S}^{\prime}} S\right|=\left|\bigcup_{S \in \mathcal{S}_{1}} S\right|-\left|\bigcup_{S \in \mathcal{S}^{\prime \prime}} S\right| \geq \frac{N}{2}-\left|\bigcup_{C \in \mathcal{C}} S_{C}\right| \geq 2^{45} \cdot 2^{n}-2^{18} \cdot 2^{n}>2^{44} \cdot 2^{n}
$$

For each $i$, let $\mathcal{S}_{i}^{\prime}=\left\{S \in \mathcal{S}^{\prime}: d(S)=i\right\}$. Suppose that $\left|\mathcal{S}_{i}^{\prime}\right| \leq 2^{14} i^{5}$ for all $i$. Then, since each set in $\mathcal{S}_{i}^{\prime}$ has size $2^{18} \cdot 2^{n-i}$, we have

$$
\left|\bigcup_{S \in \mathcal{S}^{\prime}} S\right|=\sum_{i} \sum_{S \in \mathcal{S}_{i}^{\prime}}|S| \leq \sum_{i} 2^{14} i^{5} \cdot 2^{18} \cdot 2^{n-i}=2^{32} \cdot 2^{n} \sum_{i} \frac{i^{5}}{2^{i}}<2^{44} \cdot 2^{n},
$$


which is a contradiction (we used the fact that $\sum_{i} \frac{i^{5}}{2^{2}}<2^{12}$ ). Therefore, there exists an index $i$ for which $\left|\mathcal{S}_{i}^{\prime}\right|>2^{14} i^{5}$. We have $i>0$ since otherwise the set of codimension zero would have been added to the tiling in the first step.

Let $C=\left(a_{1}, \ldots, a_{i}, *, \ldots, *\right)$. For each $\ell=1,2$, let $\mathcal{A}_{\ell}$ be the family of cubes of level $\ell$ in our partial double tiling which are adjacent to $C$ and have codimension at most $i$. By Proposition 3.2, we have $\left|\mathcal{A}_{\ell}\right| \leq i$ for each $\ell$. For a cube $A \in \mathcal{A}_{\ell}$, we say that a set $S \in \mathcal{S}_{i}^{\prime}$ is bad for $A$ if there are at least $\frac{1}{(8 i)^{5-\ell}}\left|S_{A}\right||S|$ blue edges between $S_{A}$ and $S$. Otherwise, we say that $S$ is good for $A$. We claim that there are at most $2^{13} i^{4}$ sets in $\mathcal{S}_{i}^{\prime}$ which are bad for each fixed $A$.

Let $X_{i}=\bigcup_{S \in \mathcal{S}_{i}^{\prime}} S$ and note, by Proposition [3.1, that there are at most $\left|S_{A}\right| \cdot 2^{19} \cdot 2^{n-i}$ blue edges between the sets $S_{A}$ and $X_{i}$. Each set $S \in \mathcal{S}_{i}^{\prime}$ which is bad for $A$ accounts for at least

$$
\frac{1}{(8 i)^{5-\ell}}\left|S_{A}\right||S| \geq \frac{2^{18} \cdot 2^{n-i}}{(8 i)^{4}}\left|S_{A}\right|=\frac{2^{6} \cdot 2^{n-i}}{i^{4}}\left|S_{A}\right|
$$

such blue edges (note that $|S|=2^{18} \cdot 2^{n-i}$ ). Therefore, in total, there are at most $2^{13} \cdot i^{4}$ sets in $\mathcal{S}_{i}^{\prime}$ which are in bad relation with $A$, as claimed above. Since $\left|\mathcal{A}_{1} \cup \mathcal{A}_{2}\right| \leq 2 i$ and $\left|\mathcal{S}_{i}^{\prime}\right|>2^{14} i^{5}$, there exists $S \in \mathcal{S}_{i}^{\prime}$ which is good for all cubes in $\mathcal{A}_{1} \cup \mathcal{A}_{2}$.

In order to show that $C$ satisfies (a) and (b), it suffices to verify that $i \geq d$, since $i \geq d$ implies the fact that $C$ is disjoint from all the other cubes in $\mathcal{C}_{1}$ (if $C$ intersects some other cube, then that cube must contain $C$ and therefore also contains $\left(a_{1}, \ldots, a_{n}\right)$ by Proposition 2.2). Furthermore, if this is the case, assigning $C$ to $S$ is a proper assignment (thus we have (c)).

Now suppose, for the sake of contradiction, that $i<d$ and consider the time $t$ immediately after we last embedded a cube of codimension at most $i$. At time $t$, since $\left(a_{1}, \ldots, a_{n}\right)$ was not covered, the cubes in $\mathcal{C}$ are disjoint from $C$. Moreover, the set of cubes of codimension at most $i$ which are adjacent to $C$ is the same as at current time. Therefore, $C$ could have been added to the tiling at time $t$ as well, and this contradicts the fact that we always choose a cube of minimum codimension. Thus we have $i \geq d$, as claimed.

Case 2: $\left(a_{1}, \ldots, a_{n}\right)$ is covered by a cube $C_{1} \in \mathcal{C}_{1}$ but not by a cube in $\mathcal{C}_{2}$.

In this case, in order to assign some cube $C \subset C_{1}$ containing $\left(a_{1}, \ldots, a_{n}\right)$ to a subset of $S_{C_{1}}$ in $\mathcal{S}_{2}$, there are two types of adjacency that one needs to consider. Let $C_{2}=\left(a_{1}, \ldots, a_{d}, *, \ldots, *\right)$ and temporarily consider it as a level 2 cube. We first consider the cubes which are 1-adjacent to $C_{2}$ and remove all the subsets of $S_{C_{1}}$ which are 'bad' for these cubes. The reason we consider cubes which are adjacent to $C_{2}$ instead of $C$ is because we do not know what $C$ will be at this point. Note that, depending on the choice of $C$, some of the cubes that are 1-adjacent to $C_{2}$ may not be 1-adjacent to $C$. However, since we choose $C \subset C_{2}$ (as we will show later in the proof), the set of cubes which are 1-adjacent to $C$ will be a subset of the set of cubes which are 1-adjacent to $C_{2}$. Moreover, if $C^{\prime}$ is a cube 1-adjacent to both $C$ and $C_{2}$, then $\delta\left(C^{\prime}, C\right)=\delta\left(C^{\prime}, C_{2}\right)$. Having removed all 'bad' subsets, we have enough information to determine $C$. Then, by considering the relation of the remaining subsets of $S_{C_{1}}$ to cubes which are 2-adjacent to $C$, we can find a set $S_{C}$ that can be assigned to $C$. Note that unlike in the previous case, even if $C^{\prime}$ is a cube 2-adjacent to both $C$ and $C_{2}$, we do not necessarily have $\delta\left(C^{\prime}, C\right)=\delta\left(C^{\prime}, C_{2}\right)$. For this reason, it turns out to be crucial that we have already determined $C$ before the second step.

Let $d_{1}=d\left(C_{1}\right)$ and let $\mathcal{A}^{(1)}$ be the subfamily of our partial embedding $\mathcal{C}$ consisting of cubes which are 1-adjacent to $C_{2}$. Note that, by Proposition 3.2(ii), there are at most $d_{1}$ cubes which are 1-adjacent 
to $C_{2}$ in each level and thus $\left|\mathcal{A}^{(1)}\right| \leq 2 d_{1}$. Let $\mathcal{F}=\left\{S \in \mathcal{S}_{2}: S \subset S_{C_{1}}\right\}$. By Proposition 3.1, we have $\left|\bigcup_{S \in \mathcal{F}} S\right| \geq \frac{\left|S_{C_{1}}\right|}{2}$. We say that a set $S \in \mathcal{F}$ is bad for a cube $A \in \mathcal{A}^{(1)}$ if there are at least $\left(8 \delta_{A}\right)^{\ell(S)+\ell\left(S_{A}\right)-6}|S|\left|S_{A}\right|=\frac{|S|\left|S_{A}\right|}{\left(8 \delta_{A}\right)^{4-\ell(A)}}$ blue edges between $S$ and $S_{A}$ (where $\left.\delta_{A}=\max \left\{d_{1}, d_{1}(A)\right\}\right)$. Otherwise, we say that $S$ is good for $A$. For each fixed $A$, let $\mathcal{F}_{A}$ be the subfamily of $\mathcal{F}$ consisting of sets which are bad for $A$. By the properness of the assignment up to this point, we know that there are at most $\frac{\left|S_{C_{1}}\right|\left|S_{A}\right|}{\left(8 \delta_{A}\right)^{5-\ell(A)}}$ blue edges between $S_{C_{1}}$ and $S_{A}$ for every $A \in \mathcal{A}^{(1)}$. Therefore, by counting the number of blue edges between $S_{C_{1}}$ and $S_{A}$ in two ways, we see that

$$
\sum_{S \in \mathcal{F}_{A}} \frac{|S|\left|S_{A}\right|}{\left(8 \delta_{A}\right)^{4-\ell(A)}} \leq \frac{\left|S_{C_{1}}\right|\left|S_{A}\right|}{\left(8 \delta_{A}\right)^{5-\ell(A)}}
$$

from which we have $\sum_{S \in \mathcal{F}_{A}}|S| \leq \frac{1}{8 \delta_{A}}\left|S_{C_{1}}\right| \leq \frac{1}{8 d_{1}}\left|S_{C_{1}}\right|$.

Let $\mathcal{F}^{\prime \prime}$ be the subfamily of $\mathcal{F}$ of sets which are already assigned to some cube in $\mathcal{C}_{2}$. There are no sets of relative codimension zero in $\mathcal{F}^{\prime \prime}$ since this would imply that $\left(a_{1}, \ldots, a_{n}\right)$ is covered by a cube in $\mathcal{C}_{2}$. It thus follows that for every $C \in \mathcal{C}_{2}$ such that $C \subset C_{1}$, we have $\left|S_{C}\right|=8|C|$ and

$$
\left|\bigcup_{S \in \mathcal{F}^{\prime \prime}} S\right|=\sum_{S \in \mathcal{F}^{\prime \prime}}|S| \leq 8 \sum_{C \subset C_{1}}|C| \leq 8\left|C_{1}\right|=8 \cdot 2^{n-d_{1}}
$$

Let $\mathcal{S}^{\prime}=\mathcal{F} \backslash\left(\mathcal{F}^{\prime \prime} \cup \bigcup_{A \in \mathcal{A}^{(1)}} \mathcal{F}_{A}\right)$ be the subfamily of $\mathcal{F}=\left\{S \in \mathcal{S}_{2}: S \subset S_{C_{1}}\right\}$ of sets which are not assigned to any cubes yet and are good for all the cubes in $\mathcal{A}^{(1)}$. Since $\left|S_{C_{1}}\right| \geq 2^{18}\left|C_{1}\right|=2^{18} \cdot 2^{n-d_{1}}$, we have

$$
\begin{aligned}
\left|\bigcup_{S \in \mathcal{S}^{\prime}} S\right| & \geq\left|\bigcup_{S \in \mathcal{F}} S\right|-\left|\bigcup_{S \in \mathcal{F}^{\prime \prime}} S\right|-\sum_{A \in \mathcal{A}^{(1)}}\left|\bigcup_{S \in \mathcal{F}_{A}} S\right| \\
& \geq \frac{\left|S_{C_{1}}\right|}{2}-8 \cdot 2^{n-d_{1}}-2 d_{1} \cdot \frac{\left|S_{C_{1}}\right|}{8 d_{1}}>2^{15} \cdot 2^{n-d_{1}} .
\end{aligned}
$$

For each $i \geq 0$, let $\mathcal{S}_{i}^{\prime}=\left\{S \in \mathcal{S}^{\prime}: d_{2}(S)=i\right\}=\left\{S \in \mathcal{S}^{\prime}: d(S)=d_{1}+i\right\}$. Suppose that $\left|\mathcal{S}_{i}^{\prime}\right| \leq 128 i^{3}$ for all $i$. Then, since we have $|S|=8 \cdot 2^{n-d_{1}-i}$ for all $S \in \mathcal{S}_{i}^{\prime}$,

$$
\left|\bigcup_{S \in \mathcal{S}^{\prime}} S\right|=\sum_{i} \sum_{S \in \mathcal{S}_{i}^{\prime}}|S| \leq \sum_{i} 128 i^{3} \cdot 8 \cdot 2^{n-d_{1}-i}<2^{15} \cdot 2^{n-d_{1}}
$$

which is a contradiction. Therefore, there exists an index $i$ for which $\left|\mathcal{S}_{i}^{\prime}\right|>128 \cdot i^{3}$.

Let $C=\left(a_{1}, \ldots, a_{d_{1}+i}, *, \ldots, *\right)$ and consider it as a level 2 cube. By Proposition 3.2 (ii), there are at most $i$ cubes in $\mathcal{C}$ which have level 2 adjacency with $C$ and have codimension at most $d_{1}+i$. Let $\mathcal{A}^{(2)}$ be the family consisting of these cubes. For a cube $A \in \mathcal{A}^{(2)}$, we say that a set $S \in \mathcal{S}_{i}^{\prime}$ is bad for $A$ if there are at least $(8 i)^{\ell\left(S_{A}\right)+\ell(S)-6}\left|S_{A}\right||S|=\frac{1}{(8 i)^{2}}\left|S_{A}\right||S|$ blue edges between $S_{A}$ and $S$. Otherwise, we say that $S$ is good for $A$. We claim that there are at most $128 i^{2}$ sets in $\mathcal{S}_{i}^{\prime}$ which are bad for each fixed $A$.

Let $X_{i}=\bigcup_{S \in \mathcal{S}_{i}^{\prime}} S$ and note, by Proposition 3.1, that there are at most $\left|S_{A}\right| \cdot 16 \cdot 2^{n-i}$ blue edges between the sets $S_{A}$ and $X_{i}$. Each set $S \in \mathcal{S}_{i}^{\prime}$ which is bad for $A$ accounts for at least

$$
\frac{1}{(8 i)^{2}}\left|S_{A}\right||S|=\frac{8 \cdot 2^{n-i}}{64 i^{2}}\left|S_{A}\right|=\frac{2^{n-i}}{8 i^{2}}\left|S_{A}\right|
$$


such blue edges (note that $|S|=8 \cdot 2^{n-i}$ ). Therefore, in total, there are at most $128 i^{2}$ sets in $\mathcal{S}_{i}^{\prime}$ which are bad for $A$, as claimed above. Since there are at most $i$ sets in $\mathcal{A}^{(2)}$ and $\left|\mathcal{S}_{i}^{\prime}\right|>128 i^{3}$, there exists a set $S \in \mathcal{S}_{i}^{\prime}$ which is good for all the cubes $A \in \mathcal{A}^{(2)}$.

In order to show that $C$ satisfies (a) and (b), it suffices to verify that $d_{1}+i \geq d$, since this implies the fact that $C$ is disjoint from all the other cubes in $\mathcal{C}_{2}$ (note that $C \subset C_{1}$ and if $C$ intersects some other cube of level 2 , then that cube must contain $C$ and therefore also contains $\left(a_{1}, \ldots, a_{n}\right)$ by Proposition 2.2). Furthermore, if this is the case, assigning $C$ to $S$ is a proper assignment (thus we have $(c))$.

Now suppose, for the sake of contradiction, that $d_{1}+i<d$ and consider the time $t$ immediately after we last embedded a cube of codimension at most $d_{1}+i$. At time $t$, since $d_{1}+i \geq d_{1}=d\left(C_{1}\right)$, the cube $C_{1}$ was already embedded and, since there are no cubes of level 2 covering $\left(a_{1}, \ldots, a_{n}\right)$, the cubes in $\mathcal{C}_{2}$ are disjoint from $C$. A cube in the partial embedding at time $t$, which is of codimension at most $d_{1}+i$, is 1 -adjacent to $C$ if and only if it is 1 -adjacent to $C_{2}$. Hence, the family of cubes which are adjacent to $C$ at time $t$ is a subfamily of $\mathcal{A}^{(1)} \cup \mathcal{A}^{(2)}$. Therefore, $C$ could have been added to the tiling at time $t$ as well, contradicting the fact that we always choose a cube of minimum codimension. Thus we have $d_{1}+i \geq d$, as claimed.

Note that as an outcome of our algorithm, we obtain a tiling $\mathcal{C}$ such that for every pair of adjacent cubes $C, C^{\prime} \in \mathcal{C}$, we have control on the number of blue edges between $S_{C}$ and $S_{C^{\prime}}$ (as given in the definition of proper assignment).

\subsection{Imposing a maximum degree condition}

As in the triangle case, we now impose certain maximum degree conditions between the sets $S_{C}$ for $C \in \mathcal{C}$. It suffices to impose maximum degree conditions between sets of level 2 .

For a set $C \in \mathcal{C}_{2}$ of codimension $d=d(C)$, and relative codimensions $d_{1}=d_{1}(C), d_{2}=d_{2}(C)$, recall that we have a set $S_{C} \in \mathcal{S}$ such that $\left|S_{C}\right| \geq 8 \cdot 2^{n-d}$. Let $\mathcal{A}_{\rho}$ be the family of cubes in $\mathcal{C}_{2}$ with codimension at most $d$ which have level $\rho$ adjacency with $C$. For each $A \in \mathcal{A}_{\rho}$, let $\delta_{A, \rho}=$ $\max \left\{d_{\rho}, d_{\rho}(A)\right\}$. By Proposition 3.2(ii), we have $\left|\mathcal{A}_{\rho}\right| \leq d_{\rho}$ for each $\rho=1,2$. For each $A \in \mathcal{A}_{\rho}$, there are at most $\frac{1}{64 \delta_{A, \rho}^{2}}\left|S_{C}\right|\left|S_{A}\right|$ blue edges between $S_{C}$ and $S_{A}$.

Now for $\rho=1,2$ and each $A \in \mathcal{A}_{\rho}$, remove all the vertices in $S_{C}$ which have at least $\frac{1}{8 \delta_{A, \rho}}\left|S_{A}\right|$ blue neighbors in $S_{A}$ and let $T_{C}$ be the subset of $S_{C}$ left after these removals. Since there are at most $\frac{1}{64 \delta_{A, \rho}^{2}}\left|S_{C}\right|\left|S_{A}\right|$ blue edges between $S_{C}$ and $S_{A}$, we remove at most $\frac{\left|S_{C}\right|}{8 \delta_{A, \rho}}$ vertices from $S_{C}$ for each set $A \in \mathcal{A}_{\rho}$. Thus the resulting set $T_{C}$ is of size at least

$$
\left|T_{C}\right| \geq\left|S_{C}\right|-d_{1} \cdot \frac{\left|S_{C}\right|}{8 \delta_{A, 1}}-d_{2} \cdot \frac{\left|S_{C}\right|}{8 \delta_{A, 2}} \geq \frac{\left|S_{C}\right|}{2} \geq 4 \cdot 2^{n-d}
$$

For each $A \in \mathcal{A}_{\rho}$, all the vertices in $T_{C}$ have blue degree at most $\frac{1}{8 \delta_{A, \rho}}\left|S_{A}\right| \leq \frac{1}{4 \delta_{A, \rho}}\left|T_{A}\right|$ in the set $T_{A}$. Thus we obtain the following property.

Maximum degree condition. Let $C, C^{\prime} \in \mathcal{C}_{2}$ be a pair of cubes having level $\rho$ adjacency with $d(C) \geq d\left(C^{\prime}\right)$. Then every vertex in $T_{C}$ has at most $\frac{1}{4 \delta_{\rho}}\left|T_{C^{\prime}}\right|$ blue neighbors in the set $T_{C^{\prime}}\left(\right.$ where $\left.\delta_{\rho}=\max \left\{d_{\rho}(C), d_{\rho}\left(C^{\prime}\right)\right\}\right)$. 


\subsection{Embedding the cube}

We now show how to embed $Q_{n}$. Recall that we found a double tiling $\mathcal{C}$ of $Q_{n}$. We will greedily embed the cubes in the level 2 tiling $\mathcal{C}_{2}$ one by one into their assigned sets from the family $\mathcal{S}_{2}$, in decreasing order of their codimensions. If there are several cubes of the same codimension, then we arbitrary choose the order between them.

Suppose that we are about to embed the cube $C \in \mathcal{C}_{2}$. Let $d=d(C), d_{1}=d_{1}(C)$ and $d_{2}=d_{2}(C)$. We will greedily embed the vertices of $C$ into the set $T_{C} \subseteq S_{C}$. Suppose that we are about to embed $x \in C$ and let $f: Q_{n} \rightarrow[N]$ denote the partial embedding of the cube $Q_{n}$ obtained so far. For $\rho=1,2$, let $A_{\rho}$ be the set of neighbors of $x$ which are already embedded and belong to a cube other than $C$ that has level $\rho$ adjacency with $C$. Note that we have $\left|A_{\rho}\right| \leq d_{\rho}$ for $\rho=1$ and 2 . Since we have so far only embedded cubes of codimension at least $d$, we have, for each $\rho=1$, 2 , that the vertices $f(v)$ for $v \in A_{\rho}$ have blue degree at most $\frac{1}{4 d_{\rho}}\left|T_{C}\right|$ in the set $T_{C}$, by the maximum degree condition imposed in Section 3.3. Together, these neighbors forbid at most

$$
d_{1} \cdot \frac{1}{4 d_{1}}\left|T_{C}\right|+d_{2} \cdot \frac{1}{4 d_{2}}\left|T_{C}\right| \leq \frac{1}{2}\left|T_{C}\right| .
$$

vertices of $T_{C}$ from being the image of $x$.

In addition, $x$ has at most $n-d$ neighbors which are already embedded and belong to $C$. By Proposition 3.1, for each such vertex $v, f(v)$ has blue degree at most $\frac{2^{n-d}}{n}$ in the set $T_{C}$. Together, these neighbors forbid at most $2^{n-d}$ vertices of $T_{C}$ from being the image of $x$. Finally, there are at most $2^{n-d}-1$ vertices in $T_{C}$ which are images of some other vertex of $C$ that is already embedded. Therefore, the number of vertices in $T_{C}$ into which we cannot embed $x$ is at most

$$
\frac{1}{2}\left|T_{C}\right|+2^{n-d}+\left(2^{n-d}-1\right)<\left|T_{C}\right|,
$$

where the inequality follows since $\left|T_{C}\right| \geq 4 \cdot 2^{n-d}$. Hence, there exists a vertex in $T_{C}$ which we can choose as an image of $x$ to extend the current partial embedding of the cube. Repeating this procedure until we embed the whole cube $Q_{n}$ completes the proof.

\section{General case}

In this section, we further extend the arguments presented so far to prove the main theorem. The framework is very similar to that of the previous sections, where we find multiple levels of tiling of $Q_{n}$. We begin by preprocessing the coloring to find families of sets $\mathcal{S}=\mathcal{S}_{0} \cup \mathcal{S}_{1} \cup \ldots \cup \mathcal{S}_{s-2}$ such that, for $\ell \geq 1$, sets in $\mathcal{S}_{\ell}$ are subsets of sets in $\mathcal{S}_{\ell-1}$ which do not contain blue $K_{s-\ell}$ (except for some special cases). We refer to the sets in $\mathcal{S}_{\ell}$ as level $\ell$ sets and, for a set $S \in \mathcal{S}_{\ell}$, we let its level be $\ell(S)=\ell$. Note that, unlike in the cases where $s=3,4$, we have an additional level, level zero. Level zero will consist of a single set $[N]$ and it is there merely for technical reasons.

We then seek a corresponding (multiple level) tiling $\mathcal{C}$ of the cube $Q_{n}$. Define an $(s-1)$-fold tiling (or $(s-1)$-tiling, for short) of $Q_{n}$ as a collection of $s-1$ tilings $\mathcal{C}_{0} \cup \mathcal{C}_{1} \cup \ldots \cup \mathcal{C}_{s-2}$, where $\mathcal{C}_{0}$ is the trivial tiling consisting of the unique cube $Q_{n}$, and, for all $\ell \geq 1, \mathcal{C}_{\ell}$ is a refined tiling of $\mathcal{C}_{\ell-1}$ (i.e. for every $C \in \mathcal{C}_{\ell}$, there exists $C^{\prime} \in \mathcal{C}_{\ell-1}$ such that $C \subset C^{\prime}$ ). We refer to cubes in $\mathcal{C}_{\ell}$ as level $\ell$ cubes and, for a cube $C \in \mathcal{C}_{\ell}$, define its level as $\ell(C)=\ell$. We will construct the tiling by finding cubes $C \in \mathcal{C}$ 
and assigning each of them to some set $S_{C} \in \mathcal{S}$. Informally, this means that the subcube $C$ of $Q_{n}$ will be found in the $S_{C}$ part of our graph. Note that the trivial level zero cube $Q_{n}$ gets assigned to the trivial level zero set $[N]$ and this fits the heuristic.

For the rest of this section, we assume that $s \geq 5$. Let $c=s^{15 s}$ and suppose that $N \geq c^{s} 2^{n}=s^{15 s^{2}} 2^{n}$. We will later use the following estimate.

Lemma 4.1. For every positive integer $s, \sum_{i=1}^{\infty} \frac{i^{s}}{2^{i}} \leq 2 s^{s}$.

Proof. Let $(x)_{t}=x(x-1) \ldots(x-t+1), X_{t}=\sum_{i=1}^{\infty} \frac{i^{t}}{2^{i}}$ and $Y_{t}=\sum_{i=1}^{\infty} \frac{(i)_{t}}{2^{i}}$ for non-negative integers $t$. The Stirling number $S(t, k)$ of the second kind is the number of ways to partition a set of $t$ objects into $k$ non-empty subsets. These numbers satisfy the following well-known identity $x^{t}=\sum_{k=0}^{t} S(t, k)(x)_{k}$ (see, e.g., 34, Chapter 1.4). This implies the identity

$$
X_{t}=\sum_{k=0}^{t} S(t, k) Y_{k}
$$

By taking the derivative $k$ times of both sides of the equality $(1-z)^{-1}=\sum_{i \geq 0} z^{i}$, note that

$$
k ! \cdot(1-z)^{-(k+1)}=\sum_{i \geq 1} i(i-1) \ldots(i-k+1) z^{i-k} .
$$

By multiplying both sides by $z^{k}$ and substituting $z=1 / 2$ we have that $Y_{k}=2 k$ !. This, together with the above identity, implies that $X_{t}=\sum_{k=0}^{t} 2 k ! S(t, k)$. Let $T_{s}$ be the number of partitions of a set of $s$ objects into labelled nonempty subsets. By counting over the size $k$ of the partition, we have the identity $T_{s}=\sum_{k=0}^{s} k ! S(s, k)=X_{s} / 2$. As each partition counted by $T_{s}$ is determined by the vector of labels of the sets containing each object, and there are at most $s$ such labels for each partition, we have $T_{s} \leq s^{s}$ and the desired inequality follows.

(Although it will be not be needed, we remark that there is an explicit formula

$$
X_{t}=2 \sum_{k=0}^{t} \sum_{j=0}^{k}(-1)^{k-j}\left(\begin{array}{l}
k \\
j
\end{array}\right) j^{t}
$$

which follows from substituting in the well-known identity $\left.S(t, k)=\frac{1}{k !} \sum_{j=0}^{k}(-1)^{k-j}\left(\begin{array}{c}k \\ j\end{array}\right) j^{t}.\right)$

\subsection{Preprocessing the coloring}

Let $\mathcal{S}_{0}=\{[N]\}$ and $[N]$ be the unique set of level zero and codimension zero (denoted as $\ell(S)=0$ and $d(S)=0)$. We construct the levels one at a time. Once we finish constructing $\mathcal{S}_{\ell-1}$, for each set $S^{\prime} \in \mathcal{S}_{\ell-1}$, we use the following procedure to construct sets belonging to the $\ell$-th level $\mathcal{S}_{\ell}$ :

1. If $S^{\prime}$ is not exceptional: for each $d=0,1,2, \ldots, \log n+s \log c$, if there exists a set $S \subset S^{\prime}$ which induces a blue $K_{s-\ell}$-free graph of order exactly $c^{s-\ell} \cdot 2^{n-d\left(S^{\prime}\right)-d}$, then arbitrarily choose one, add it to the family $\mathcal{S}_{\ell}$ and remove the vertices of $S$ from $S^{\prime}$. We define the $\ell$-codimension $d_{\ell}(S)$ of such a set as $d_{\ell}(S)=d$ and, for $i=1, \ldots, \ell-1$, we define the $i$-codimension $d_{i}(S)$ as $d_{i}(S)=d_{i}\left(S^{\prime}\right)$. Let the codimension of $S$ be $d(S)=\sum_{i=1}^{\ell} d_{i}(S)$. 
When there are no more such sets, continue to the next value of $d$. If, after running through all values of $d, \sum_{S \in \mathcal{S}_{\ell}, S \subset S^{\prime}}|S|<\frac{\left|S^{\prime}\right|}{2}$, then add the set of remaining vertices to $\mathcal{S}_{\ell}$ and declare it to be an exceptional set with $\ell$-codimension zero (note that this set has size at least $\left.\frac{\left|S^{\prime}\right|}{2}=\frac{c^{s-(\ell-1)} \cdot 2^{n-d\left(S^{\prime}\right)}}{2} \geq c^{s-\ell} \cdot 2^{n-d\left(S^{\prime}\right)}\right)$.

2. If $S^{\prime}$ is exceptional: add $S^{\prime}$ to the family $\mathcal{S}_{\ell}$ and define its $\ell$-codimension as zero.

Let $\mathcal{S}=\bigcup_{\ell=0}^{s-2} \mathcal{S}_{\ell}$ (we suppose that $\mathcal{S}$ is a multi-family and if a set appears multiple times we distinguish them by their levels). The following proposition is similar to Proposition 3.1. We omit its proof.

Proposition 4.1. (i) For $1 \leq \ell \leq s-2$ and a set $S^{\prime} \in \mathcal{S}_{\ell-1}, \sum_{S \in \mathcal{S}_{\ell}, S \subset S^{\prime}}|S| \geq \frac{\left|S^{\prime}\right|}{2}$.

(ii) For integers $1 \leq \ell \leq s-2$ and $i \geq 1$, let $S^{\prime}$ be a set of level $\ell-1$ and let

$$
X=\bigcup_{S \in \mathcal{S}_{\ell}, S \subset S^{\prime}, d_{\ell}(S) \geq i} S
$$

Then each vertex $v \in S^{\prime}$ has at most $2 c^{s-\ell} \cdot 2^{n-d\left(S^{\prime}\right)-i}$ blue neighbors in $X$.

(iii) For every set $S \in \mathcal{S}_{s-2}$, the subgraph induced by $S$ has maximum blue degree at most $\frac{2^{n-d(S)}}{n}$.

\subsection{Tiling the cube}

In this subsection, we find an $(s-1)$-tiling of $Q_{n}$. Recall that in the previous section, we had to control the blue edge densities between adjacent cubes in the tiling. The parameter that governed the control of these densities was defined in terms of the $\rho$-codimension, where $\rho$ was the level of adjacency of these cubes. Below we generalize this concept.

Definition 4.1. Let $\mathcal{C}$ be an $(s-1)$-tiling and let $C, C^{\prime} \in \mathcal{C}$ be two adjacent cubes.

(i) The level of adjacency $\rho\left(C, C^{\prime}\right)$ is the minimum $\ell$ such that the cubes of level $\ell$ containing $C$ and $C^{\prime}$ are distinct. We say that $C$ and $C^{\prime}$ are $\rho$-adjacent if $\rho\left(C, C^{\prime}\right)=\rho$.

(ii) The dominating parameter $\delta\left(C, C^{\prime}\right)$ is $\max \left\{d_{\rho}(C), d_{\rho}\left(C^{\prime}\right)\right\}$, where $\rho=\rho\left(C, C^{\prime}\right)$.

Note that the level of adjacency of two cubes $C$ and $C^{\prime}$ is at most $\min \left\{\ell(C), \ell\left(C^{\prime}\right)\right\}$. The following proposition is similar to Proposition 3.2 and we omit its proof.

Proposition 4.2. Let $\mathcal{C}$ be an $(s-1)$-tiling and let $C \in \mathcal{C}$ be a level $\ell$ special cube of codimension $d$. For each $\rho=1,2, \ldots, \ell$ and $\ell^{\prime}=1,2, \ldots, s-2$, the number of special cubes of level $\ell^{\prime}$ and codimension at most $d$ which are $\rho$-adjacent to $C$ is at most $d_{\rho}(C)$.

Our $(s-1)$-tiling $\mathcal{C}$ of the cube $Q_{n}$ will be constructed in correspondence with the family $\mathcal{S}$ constructed in Section 4.1. We will construct $\mathcal{C}$ by finding cubes of the tiling one by one. We slightly abuse notation and use $\mathcal{C}$ also to denote the 'partial' $(s-1)$-tiling, where only part of the cube $Q_{n}$ is covered. At each step, we find a subcube $C$ which covers some non-covered part of $Q_{n}$ and assign it to some set $S_{C} \in \mathcal{S}$. We say that such an assignment is proper if the following properties hold.

\section{Proper assignment.}

1. $\ell(C)=\ell\left(S_{C}\right)$ and, for $\ell=\ell(C)$, we have $d_{\ell}(C)=d_{\ell}\left(S_{C}\right)$. 
2. If $C \subset C^{\prime}$ for $C^{\prime} \in \mathcal{C}$, then $S_{C} \subset S_{C^{\prime}}$.

3. Suppose that $C$ is adjacent to some cube $C^{\prime}$ already in the tiling, where $C^{\prime}$ is assigned to $S_{C^{\prime}}$, and that $C$ and $C^{\prime}$ have level $\rho$ adjacency. Then the number of blue edges in the bipartite graph induced by $S_{C}$ and $S_{C^{\prime}}$ is at most $\left|S_{C} \| S_{C^{\prime}}\right| \cdot\left(4 s^{2} \delta\left(C, C^{\prime}\right)\right)^{\ell(C)+\ell\left(C^{\prime}\right)-2 s}$.

We use $S_{C}$ to denote the set in $\mathcal{S}$ to which $C$ is assigned. Our algorithm for finding the tiling $\mathcal{C}$ and the corresponding sets in $\mathcal{S}$ is as follows.

Tiling Algorithm. At each step, consider all possible cubes $C$ which

(a) can be added to $\mathcal{C}$ to extend the partial tiling,

(b) have $d(C) \geq d\left(C^{\prime}\right)$ for all $C^{\prime} \in \mathcal{C}$, and

(c) for which there exists a set $S \in \mathcal{S}$ which has not yet been assigned and such that assigning $C$ to $S$ gives a proper assignment.

Take a cube $C_{0}$ of minimum codimension satisfying (a), (b) and (c) and add it to the tiling. Assign $C_{0}$ to the set $S_{C_{0}} \in \mathcal{S}$ given by (c).

The following proposition shows that the algorithm will terminate successfully.

Proposition 4.3. If the tiling is not complete, then the algorithm always chooses a cube from a non-empty collection.

Proof. In the beginning, the algorithm will take $Q_{n}$ as the level 0 tiling and will assign it to $[N]$, which is the unique set of level 0 . Now suppose that in the previous step we embedded some cube of codimension $d$. Since the tiling is not complete, there exists a vertex $\left(a_{1}, \ldots, a_{n}\right) \in Q_{n}$ which is not covered $s-1$ times. Suppose that this vertex is covered $\ell$ times for $\ell \leq s-2$ and let $C_{0}, \ldots, C_{\ell-1}$ be the cubes of each level that cover it (note that $\left.C_{0} \supseteq C_{1} \supseteq \ldots \supseteq C_{\ell-1}\right)$. Let $C_{\ell}=\left(a_{1}, \ldots, a_{d}, *, \ldots, *\right)$. We temporarily consider $C_{\ell}$ as a level $\ell$ cube.

In this case, in order to assign some cube $C \subset C_{\ell-1}$ containing $\left(a_{1}, \ldots, a_{n}\right)$ to a subset of $S_{C_{\ell-1}}$ in $\mathcal{S}_{\ell}$, we first consider the cubes which are $\rho$-adjacent to $C_{\ell}$ for $\rho \leq \ell-1$ and remove all the subsets of $S_{C_{\ell-1}}$ which are 'bad' for these cubes. The reason we consider cubes which are adjacent to $C_{\ell}$ instead of $C$ is because we do not know what $C$ will be at this point. Note that, depending on the choice of $C$, some of the cubes that are $\rho$-adjacent to $C_{\ell}$ may not be $\rho$-adjacent to $C$. However, since we choose $C \subset C_{\ell}$ (as we will show later in the proof), the set of cubes which are $\rho$-adjacent to $C$ will be a subset of the set of cubes which are $\rho$-adjacent to $C_{\ell}$. Moreover, for $\rho \leq \ell-1$, if $C^{\prime}$ is a cube $\rho$-adjacent to both $C$ and $C_{\ell}$, then $\delta\left(C^{\prime}, C\right)=\delta\left(C^{\prime}, C_{\ell}\right)$. Having removed these 'bad' subsets, we have enough information to determine $C$. Then, by considering the relation of the remaining subsets of $S_{C_{\ell-1}}$ to cubes which are $\ell$-adjacent to $C$, we can find a set $S_{C}$ that can be assigned to $C$. Note that unlike in the previous case, for $\rho \geq \ell$, even if $C^{\prime}$ is a cube $\rho$-adjacent to both $C$ and $C_{\ell}$, we do not necessarily have $\delta\left(C^{\prime}, C\right)=\delta\left(C^{\prime}, C_{\ell}\right)$. For this reason, it turns out to be crucial that we have already determined $C$ before the second step.

For each $\rho \leq \ell-1$ and $i$, let $\mathcal{A}_{i}^{(\rho)}$ be the family of level $i$ cubes from our partial embedding $\mathcal{C}$ consisting of cubes which are $\rho$-adjacent to $C_{\ell}$. Let $\mathcal{A}^{(\rho)}=\bigcup_{i} \mathcal{A}_{i}^{(\rho)}$. By Proposition 4.2, we have $\left|\mathcal{A}_{i}^{(\rho)}\right| \leq d_{\rho}\left(C_{\ell-1}\right)$ for each $i$ and thus $\left|\mathcal{A}^{(\rho)}\right| \leq s \cdot d_{\rho}\left(C_{\ell-1}\right)$. Let $\mathcal{F}=\left\{S \in \mathcal{S}_{\ell}: S \subset S_{C_{\ell-1}}\right\}$. By 
Proposition 4.1, we have $\left|\bigcup_{S \in \mathcal{F}} S\right| \geq \frac{\left|S_{C_{\ell-1}}\right|}{2}$. We say that a set $S \in \mathcal{F}$ is bad for a cube $A \in \mathcal{A}_{i}^{(\rho)}$ if there are at least $\left(4 s^{2} \delta_{A}\right)^{\ell(S)+\ell\left(S_{A}\right)-2 s}|S|\left|S_{A}\right|=\left(4 s^{2} \delta_{A}\right)^{\ell+i-2 s}|S|\left|S_{A}\right|$ blue edges between $S$ and $S_{A}$ (where $\delta_{A}=\delta\left(C_{\ell-1}, A\right)$ is the dominating parameter of $C_{\ell-1}$ and $A$ ). Otherwise, we say that $S$ is good for $A$. For each fixed $A$, let $\mathcal{F}_{A}$ be the subfamily of $\mathcal{F}$ consisting of sets which are bad for $A$. By the properness of the assignment up to this point, we know that there are at most $\left(4 s^{2} \delta_{A}\right)^{\ell+i-1-2 s}\left|S_{C_{\ell-1}}\right|\left|S_{A}\right|$ blue edges between $S_{C_{\ell-1}}$ and $S_{A}$ for every $A \in \mathcal{A}_{i}^{(\rho)}$. Therefore, by counting the number of blue edges between $S_{C_{\ell-1}}$ and $S_{A}$ in two ways, we see that

$$
\sum_{S \in \mathcal{F}_{A}}\left(4 s^{2} \delta_{A}\right)^{\ell+i-2 s}|S|\left|S_{A}\right| \leq\left(4 s^{2} \delta_{A}\right)^{\ell+i-1-2 s}\left|S_{C_{\ell-1}}\right|\left|S_{A}\right|,
$$

from which we have $\sum_{S \in \mathcal{F}_{A}}|S| \leq \frac{1}{4 s^{2} \delta_{A}}\left|S_{C_{\ell-1}}\right| \leq \frac{1}{4 s^{2} d_{\rho}\left(C_{\ell-1}\right)}\left|S_{C_{\ell-1}}\right|$.

Let $\mathcal{F}^{\prime \prime}$ be the subfamily of $\mathcal{F}$ of sets which are already assigned to some cube in $\mathcal{C}_{\ell}$. There can be no sets of relative codimension zero in $\mathcal{F}^{\prime \prime}$ since this would imply that $\left(a_{1}, \ldots, a_{n}\right)$ is covered by a cube in $\mathcal{C}_{\ell}$. It thus follows that for every $C \in \mathcal{C}_{\ell}$ such that $C \subset C_{\ell-1}$, we have $\left|S_{C}\right|=c^{s-\ell}|C|$ and

$$
\left|\bigcup_{S \in \mathcal{F}^{\prime \prime}} S\right|=\sum_{S \in \mathcal{F}^{\prime \prime}}|S| \leq c^{s-\ell} \cdot\left|C_{\ell-1}\right|
$$

Let $\mathcal{S}^{\prime}=\mathcal{F} \backslash\left(\mathcal{F}^{\prime \prime} \cup \bigcup_{\rho} \bigcup_{A \in \mathcal{A}^{(\rho)}} \mathcal{F}_{A}\right)$ be the subfamily of $\mathcal{F}$ of sets which are not assigned to any cubes yet and are good for all the cubes in $\mathcal{A}^{(\rho)}$ for all $\rho \leq \ell-1$. Since $\left|S_{C_{\ell-1}}\right| \geq c^{s-\ell+1}\left|C_{\ell-1}\right|$, we have

$$
\begin{aligned}
\left|\bigcup_{S \in \mathcal{S}^{\prime}} S\right| & \geq\left|\bigcup_{S \in \mathcal{F}} S\right|-\left|\bigcup_{S \in \mathcal{F}^{\prime \prime}} S\right|-\sum_{\rho} \sum_{A \in \mathcal{A}^{(\rho)}}\left|\bigcup_{S \in \mathcal{F}_{A}} S\right| \\
& \geq \frac{\left|S_{C_{\ell-1}}\right|}{2}-c^{s-\ell} \cdot\left|C_{\ell-1}\right|-\sum_{\rho} s d_{\rho}\left(C_{\ell-1}\right) \cdot \frac{\mid S_{C_{\ell-1} \mid}}{4 s^{2} d_{\rho}\left(C_{\ell-1}\right)}>\frac{c^{s-\ell+1}}{5} \cdot\left|C_{\ell-1}\right| .
\end{aligned}
$$

For each $i \geq 0$, let $\mathcal{S}_{i}^{\prime}=\left\{S \in \mathcal{S}^{\prime}: d_{\ell}(S)=i\right\}=\left\{S \in \mathcal{S}^{\prime}: d(S)=d\left(C_{\ell-1}\right)+i\right\}$. Suppose that $\left|\mathcal{S}_{i}^{\prime}\right| \leq\left(4 s^{2} i\right)^{2 s+1}$ for all $i$. Then, since we have $|S|=c^{s-\ell} 2^{n-d\left(C_{\ell-1}\right)-i}=c^{s-\ell} \cdot 2^{-i}\left|C_{\ell-1}\right|$ for all $S \in \mathcal{S}_{i}^{\prime}$,

$$
\left|\bigcup_{S \in \mathcal{S}^{\prime}} S\right|=\sum_{i} \sum_{S \in \mathcal{S}_{i}^{\prime}}|S| \leq \sum_{i}\left(4 s^{2} i\right)^{2 s+1} \cdot c^{s-\ell} \cdot 2^{-i}\left|C_{\ell-1}\right| .
$$

By Lemma 4.1, we have

$$
\sum_{i}\left(4 s^{2} i\right)^{2 s+1} 2^{-i}=(2 s)^{4 s+2} \sum_{i} i^{2 s+1} / 2^{i} \leq(2 s)^{4 s+2} \cdot 2(2 s+1)^{2 s+1}<s^{15 s} / 5=c / 5 .
$$

Therefore, $\left|\bigcup_{S \in \mathcal{S}^{\prime}} S\right|<\frac{c^{s-\ell+1}}{5} \cdot\left|C_{\ell-1}\right|$, which is a contradiction. Thus there exists an index $i$ for which $\left|\mathcal{S}_{i}^{\prime}\right|>\left(4 s^{2} i\right)^{2 s+1}$.

Let $C=\left(a_{1}, \ldots, a_{d\left(C_{\ell-1}\right)+i}, *, \ldots, *\right)$ and consider it as a level $\ell$ cube. By Proposition 4.2 , there are at most $s \cdot i$ cubes in $\mathcal{C}$ with codimension at most $d(C)$ which have level $\ell$ adjacency with $C$ (remember that these may have level higher than $\ell$ ). Let $\mathcal{A}^{(\ell)}$ be the family consisting of these cubes and note that the dominating parameter of $C$ and $A$ for $A \in \mathcal{A}^{(\ell)}$ is always $i$. For a cube $A \in \mathcal{A}^{(\ell)}$, we say that a set $S \in \mathcal{S}_{i}^{\prime}$ is $b a d$ for $A$ if there are at least $\left(4 s^{2} i\right)^{\ell\left(S_{A}\right)+\ell(S)-2 s}\left|S_{A}\right||S|=\left(4 s^{2} i\right)^{\ell\left(S_{A}\right)+\ell-2 s}\left|S_{A}\right||S|$ 
blue edges between $S_{A}$ and $S$. Otherwise, we say that $S$ is good for $A$. We claim that there are at most $\left(4 s^{2} i\right)^{2 s}$ sets in $\mathcal{S}_{i}^{\prime}$ which are bad for each fixed $A$.

Let $X_{i}=\bigcup_{S \in \mathcal{S}_{i}^{\prime}} S$ and note, by Proposition 4.1, that there are at most $\left|S_{A}\right| \cdot 2 c^{s-\ell} \cdot 2^{n-d(C)}$ blue edges between the sets $S_{A}$ and $X_{i}$. Each set $S \in \mathcal{S}_{i}^{\prime}$ which is bad for $A$ accounts for at least $\left(4 s^{2} i\right)^{\ell\left(S_{A}\right)+\ell-2 s}\left|S_{A}\right||S| \geq \frac{c^{s-\ell} \cdot 2^{n-d(C)}}{\left(4 s^{2} i\right)^{2 s-1}}\left|S_{A}\right|$ such blue edges (note that $|S|=c^{s-\ell} \cdot 2^{n-d(C)}$ and $\ell \geq 1$ ). Therefore, in total, there are at most $\left(4 s^{2} i\right)^{2 s}$ sets in $\mathcal{S}_{i}^{\prime}$ which are bad for $A$, as claimed above. Since there are at most si cubes in $\mathcal{A}^{(\ell)}$ and $\left|\mathcal{S}_{i}^{\prime}\right|>\left(4 s^{2} i\right)^{2 s+1}$, there exists a set $S \in \mathcal{S}_{i}^{\prime}$ which is good for all the cubes $A \in \mathcal{A}^{(\ell)}$.

In order to show that $C$ satisfies (a) and (b), it suffices to verify that $d(C) \geq d$, since this implies the fact that $C$ is disjoint from all the other cubes of level at least $\ell$ (note that $C \subset C_{\ell-1}$ and if $C$ intersects some other cube of level at least $\ell$, then that cube must contain $C$ and therefore also contains $\left(a_{1}, \ldots, a_{n}\right)$ by Proposition 2.2). Furthermore, if this is the case, assigning $C$ to $S$ is a proper assignment (thus we have (c)).

Now suppose, for the sake of contradiction, that $d(C)<d$ and consider the time $t$ immediately after we last embedded a cube of codimension at most $d(C)$. At time $t$, since $d(C) \geq d\left(C_{\ell-1}\right)$, the cube $C_{\ell-1}$ was already embedded and, since there are no cubes of level $\ell$ covering $\left(a_{1}, \ldots, a_{n}\right)$, the cubes in $\mathcal{C}_{\ell}$ are disjoint from $C$. For $\rho \leq \ell-1$, a cube in the partial tiling at time $t$, which is of codimension at most $d(C)$, is $\rho$-adjacent to $C$ if and only if it is $\rho$-adjacent to $C_{\ell}$. Hence, the family of cubes which are adjacent to $C$ at time $t$ is a subfamily of $\bigcup_{\rho=1}^{\ell} \mathcal{A}^{(\rho)}$. Therefore, $C$ could have been added to the tiling at time $t$ as well, contradicting the fact that we always choose a cube of minimum codimension. Thus we have $d(C) \geq d$, as claimed.

Note that as an outcome of our algorithm, we obtain a tiling $\mathcal{C}$ such that for every pair of adjacent cubes $C, C^{\prime} \in \mathcal{C}$, we have control on the number of blue edges between $S_{C}$ and $S_{C^{\prime}}$ (as given in the definition of proper assignment).

\subsection{Imposing a maximum degree condition}

As in the previous sections, we now impose certain maximum degree conditions between the sets $S_{C}$ for $C \in \mathcal{C}_{s-2}$. For a set $C \in \mathcal{C}_{s-2}$ of codimension $d=d(C)$ and relative codimensions $d_{\ell}=d_{\ell}(C), 1 \leq$ $\ell \leq s-2$, recall that we have a set $S_{C} \in \mathcal{S}$ such that $\left|S_{C}\right| \geq c^{2} \cdot 2^{n-d}$. Let $\mathcal{A}^{(\rho)}$ be the family of cubes in $\mathcal{C}_{s-2}$ with codimension at most $d$ which have level $\rho$ adjacency with $C$. By Proposition 4.2, we have $\left|\mathcal{A}^{(\rho)}\right| \leq d_{\rho}(C)$ for each $\rho=1, \ldots, s-2$. For each $A \in \mathcal{A}^{(\rho)}$, let $\delta_{A}=\delta(C, A)$ and note that $\delta_{A}=\max \left\{d_{\rho}(A), d_{\rho}(C)\right\} \geq d_{\rho}(C)$. Also, since $\ell(A)+\ell(C)-2 s=-4$, there are at most $\frac{1}{\left(4 s^{2} \delta_{A}\right)^{4}}\left|S_{C}\right|\left|S_{A}\right|$ blue edges between $S_{C}$ and $S_{A}$.

Now for $\rho=1, \ldots, s-2$, and each $A \in \mathcal{A}^{(\rho)}$, remove all the vertices in $S_{C}$ which have at least $\frac{1}{4 s^{2} \delta_{A}}\left|S_{A}\right|$ blue neighbors in $S_{A}$ and let $T_{C}$ be the subset of $S_{C}$ left after these removals. Since there are at most $\frac{1}{\left(4 s^{2} \delta_{A}\right)^{4}}\left|S_{C}\right|\left|S_{A}\right|$ blue edges between $S_{C}$ and $S_{A}$, we remove at most $\frac{\left|S_{C}\right|}{\left(4 s^{2} \delta_{A}\right)^{3}}$ vertices from $S_{C}$ for each set $A \in \mathcal{A}^{(\rho)}$. Thus the resulting set $T_{C}$ is of size at least

$$
\left|T_{C}\right| \geq\left|S_{C}\right|-\sum_{\rho=1}^{s-2} d_{\rho} \cdot \frac{\left|S_{C}\right|}{\left(4 s^{2} \delta_{A}\right)^{3}} \geq \frac{\left|S_{C}\right|}{2} \geq c \cdot 2^{n-d} .
$$

For each $A \in \mathcal{A}^{(\rho)}$, all the vertices in $T_{C}$ have blue degree at most $\frac{1}{4 s^{2} \delta_{A}}\left|S_{A}\right| \leq \frac{1}{2 s^{2} \delta_{A}}\left|T_{A}\right|$ in the set 
$T_{A}$. Thus we obtain the following property.

Maximum degree condition. Let $C, C^{\prime} \in \mathcal{C}_{s-2}$ be a pair of cubes having level $\rho$ adjacency with $d(C) \geq d\left(C^{\prime}\right)$. Then every vertex in $T_{C}$ has at most $\frac{1}{2 s^{2} \delta\left(C, C^{\prime}\right)}\left|T_{C^{\prime}}\right|$ blue neighbors in the set $T_{C^{\prime}}$.

\subsection{Embedding the cube}

We now show how to embed $Q_{n}$. Recall that we found an $(s-1)$-tiling $\mathcal{C}$ of $Q_{n}$. We will greedily embed the cubes in the level $s-2$ tiling $\mathcal{C}_{s-2}$ one by one into their assigned sets from the family $\mathcal{S}_{s-2}$, in decreasing order of their codimensions. If there are several cubes of the same codimension, then we arbitrary choose the order between them.

Suppose that we are about to embed the cube $C \in \mathcal{C}_{s-2}$. Let $d=d(C)$ and $d_{\ell}=d_{\ell}(C)$, for $\ell=1, \ldots, s-2$. We will greedily embed the vertices of $C$ into $T_{C} \subseteq S_{C}$. Suppose that we are about to embed $x \in C$ and let $f: Q_{n} \rightarrow[N]$ denote the partial embedding of the cube $Q_{n}$ obtained so far. For each $\rho$, let $A_{\rho}$ be the set of neighbors of $x$ which are already embedded and belong to a cube other than $C$ that has level $\rho$ adjacency with $C$. Note that we have $\left|A_{\rho}\right| \leq d_{\rho}$ for every $\rho$. Since we have so far only embedded cubes of codimension at least $d$, for each $\rho$, the vertices $f(v)$ for $v \in A_{\rho}$ have blue degree at most $\frac{1}{2 s^{2} d_{\rho}}\left|T_{C}\right|$ in the set $T_{C}$, by the maximum degree condition imposed in Section 4.3. Together, these neighbors forbid at most

$$
\sum_{\rho=1}^{s-2} d_{\rho} \cdot \frac{1}{2 s^{2} d_{\rho}}\left|T_{C}\right| \leq \frac{1}{2}\left|T_{C}\right| .
$$

vertices of $T_{C}$ from being the image of $x$.

In addition, $x$ has at most $n-d$ neighbors which are already embedded and belong to $C$. By Proposition 4.1, for each such vertex $v, f(v)$ has blue degree at most $\frac{2^{n-d}}{n}$ in the set $T_{C}$. Together, these neighbors forbid at most $2^{n-d}$ vertices of $T_{C}$ from being the image of $x$. Finally, there are at most $2^{n-d}-1$ vertices in $T_{C}$ which are images of some other vertex of $C$ that is already embedded. Therefore, the number of vertices in $T_{C}$ into which we cannot embed $x$ is at most

$$
\frac{1}{2}\left|T_{C}\right|+2^{n-d}+\left(2^{n-d}-1\right)<\left|T_{C}\right|,
$$

where the inequality follows since $\left|T_{C}\right| \geq c \cdot 2^{n-d}$. Hence, there exists a vertex in $T_{C}$ which we can choose as an image of $x$ to extend the current partial embedding of the cube. Repeating this procedure until we embed the whole cube $Q_{n}$ completes the proof.

\section{Small separators, forbidden minors and Ramsey goodness}

Let $\mathcal{G}_{H}$ be the family of graphs $G$ which do not contain an $H$-minor. In order to show that this family is $s$-good, we wish to apply the following result of Nikiforov and Rousseau [30].

Theorem 5.1. For every $s \geq 3, d \geq 1$ and $0<\gamma<1$, there exists $\eta>0$ such that the class $\mathcal{G}$ of $d$-degenerate graphs $G$ with a $\left(|V(G)|^{1-\gamma}, \eta\right)$-separator is s-good. 
There are two conditions here that need to be verified in order to gain the conclusion of Theorem 1.2. Firstly, we need to show that the graphs in $\mathcal{G}_{H}$ have bounded degeneracy. This was first proved by Mader [28. Later, Kostochka [24, 25] and Thomason [35] independently established the following bound, which is tight apart from the constant factor. More recently, the asymptotic value of $c$ was determined by Thomason [36].

Theorem 5.2. There exists a constant $c>0$ such that any graph with average degree at least ch $\sqrt{\log h}$ contains a $K_{h}$-minor.

Secondly, we need to show that the graphs in $\mathcal{G}_{H}$ have appropriate separators. We will use the following result of Alon, Seymour and Thomas [2]. Note that, in the particular case of planar graphs, such a separator theorem was proved much earlier by Lipton and Tarjan [27].

Theorem 5.3. Let $G$ be a graph on $n$ vertices containing no $K_{h}$-minor. Then $G$ has an $\left(h^{3 / 2} n^{1 / 2}, \frac{2}{3}\right)$ separator.

We are now ready to prove our main result about $\mathcal{G}_{H}$, which we recall from the introduction.

Theorem 5.4. For every fixed graph $H$, the class $\mathcal{G}_{H}$ of graphs $G$ which do not contain an $H$-minor is $s$-good for all $s \geq 3$.

Proof. In order to apply Theorem 5.1, it is enough to verify that, for any $\eta>0$, any sufficiently large graph $G$ in $\mathcal{G}_{H}$ has bounded degeneracy and a $\left(|V(G)|^{2 / 3}, \eta\right)$-separator.

Suppose that the graph $H$ has $h$ vertices. Note, by Theorem 5.2, that any graph with average degree at least $c h \sqrt{\log h}$ contains a $K_{h}$-minor and, hence, an $H$-minor. This implies that the average degree of every subgraph of $G$ is at most $c h \sqrt{\log h}$. In turn, this easily implies that the degeneracy of $G$ is at most $c h \sqrt{\log h}$.

Suppose that $G$ has $n$ vertices. To show that a sufficiently large graph $G$ from the class $\mathcal{G}_{H}$ contains an $\left(n^{2 / 3}, \eta\right)$-separator $T$, we begin by applying Theorem 5.3 to conclude that there is an $\left(h^{3 / 2} n^{1 / 2}, \frac{2}{3}\right)$ separator. Note, by taking unions of small components if necessary, that this gives a decomposition of the vertex set of $G$ into three sets $T, A$ and $B$ such that $|T| \leq h^{3 / 2} n^{1 / 2},|A|,|B| \leq \frac{2}{3} n$ and there are no edges between $A$ and $B$.

We will prove, by induction on $i$, that for $n$ sufficiently large depending on $i$, there is a separator $T_{i}$ of size at most $2^{i} h^{3 / 2} n^{1 / 2}$ that splits the vertex set of $G$ into $2^{i}$ sets $U_{i, 1}, \ldots, U_{i, 2^{i}}$, each of size at most $\left(\frac{2}{3}\right)^{i} n$, so that there are no edges between any distinct sets $U_{i, a}$ and $U_{i, b}$. By the previous paragraph, the result holds for $i=1$. Now suppose that it holds for $i$. We will show that a similar conclusion follows for $i+1$.

To begin, we apply Theorem 5.3 within each of the sets $U_{i, j}$ to conclude that there is a decomposition of $U_{i, j}$ into sets $T_{i, j}, A_{i, j}$ and $B_{i, j}$ such that $\left|T_{i, j}\right| \leq h^{3 / 2}\left|U_{i, j}\right|^{1 / 2}$,

$$
\left|A_{i, j}\right|,\left|B_{i, j}\right| \leq \frac{2}{3}\left|U_{i, j}\right| \leq\left(\frac{2}{3}\right)^{i+1} n
$$

and there are no edges between $A_{i, j}$ and $B_{i, j}$. We let the collection $\left\{U_{i+1, j}\right\}_{j=1}^{2^{i+1}}$ consist of all sets of the form $A_{i, j}$ and $B_{i, j}$ for $j=1, \ldots, 2^{i}$. This collection has size $2^{i+1}$, each of the sets has size at 
most $\left(\frac{2}{3}\right)^{i+1} n$ and there are no edges between distinct sets $U_{i+1, a}$ and $U_{i+1, b}$. If we also let $T_{i+1}$ be the union of $T_{i}$ and $T_{i, j}$, for $1 \leq j \leq 2^{i}$, we have

$$
\left|T_{i+1}\right| \leq\left|T_{i}\right|+\sum_{j=1}^{2^{i}}\left|T_{i, j}\right| \leq 2^{i} h^{3 / 2} n^{1 / 2}+\sum_{j=1}^{2^{i}} h^{3 / 2}\left|U_{i, j}\right|^{1 / 2} \leq 2^{i} h^{3 / 2} n^{1 / 2}+2^{i} h^{3 / 2} n^{1 / 2}=2^{i+1} h^{3 / 2} n^{1 / 2} .
$$

Therefore, the induction holds.

If we now apply this result with $i=2 \log \eta^{-1}$ and $n \geq h^{9} \eta^{-12}$, we see, since $\left(\frac{2}{3}\right)^{i} \leq \eta$ and $n^{2 / 3} \geq$ $2^{i} h^{3 / 2} n^{1 / 2}$, that $G$ has an $\left(n^{2 / 3}, \eta\right)$-separator, as required. The result follows.

Let $\mathcal{K}$ be the collection of graphs $K$ for which there is a proper vertex coloring in $\chi(K)$ colors such that at least two of the color classes have size one. The full result of Nikiforov and Rousseau (namely, Theorem 2.2 of [30]) says that for any $K \in \mathcal{K}, d \geq 1$ and $0<\gamma<1$ there exists $\eta>0$ such that the class $\mathcal{G}$ of $d$-degenerate graphs $G$ with a $\left(|V(G)|^{1-\gamma}, \eta\right)$-separator is $K$-good. This may in turn be used to show that for any $H$ the family of graphs $\mathcal{G}_{H}$ is $K$-good for all $K \in \mathcal{K}$. However, $\mathcal{G}_{H}$ is not $K$-good for all graphs $K$. This follows from the observation mentioned in the introduction that $K_{1, t}$ is not $K_{2,2}$-good for any $t$.

The family of graphs $\mathcal{G}_{H}$ with forbidden $H$-minor is not the only class of graphs known to have small separators. For example, several geometric separator theorems are known (see, for example, [19, 29]) saying that the class of intersection graphs formed by certain collections of bodies have small separators. In any of these cases, Theorem 5.1 will also allow us to show that the classes are $K$-good for any $K \in \mathcal{K}$, though in some cases we may have to further restrict the class in order to obtain the required degeneracy condition.

\section{Concluding remarks}

The question of determining whether the cube is $s$-good for any $s \geq 3$ is only one of two well-known questions of Burr and Erdös regarding Ramsey numbers and the cube. The other [10, 14] is the question of determining whether the Ramsey number $r\left(Q_{n}\right):=r\left(Q_{n}, Q_{n}\right)$ of the cube with itself is linear in the number of vertices in $Q_{n}$.

Beginning with Beck [3, who proved that $r\left(Q_{n}\right) \leq 2^{c n^{2}}$, a large number of papers [22, 23, 26, 32, 33. have considered this question, with the current best bound being $r\left(Q_{n}\right) \leq n 2^{2 n+5}$, due to Fox and Sudakov [20]. That is, $r\left(Q_{n}\right) \leq\left|Q_{n}\right|^{2+o(1)}$. This (almost) quadratic bound for $r\left(Q_{n}\right)$ was obtained using a careful application of dependent random choice [21]. It seems likely that in order to improve the bound one will have to improve this latter technique. However, we do not rule out the possibility that some of the embedding lemmas used in the current paper could also be of use.

Suppose $H$ is an $N$-vertex graph with chromatic number $r$, maximum degree $O(1)$ and bandwidth $o(N)$. Bollobás and Komlós conjectured that every $N$-vertex graph $G$ which does not contain $H$ as a subgraph has minimum degree at most $\left(1-\frac{1}{r}+o(1)\right) N$. This conjecture was recently verified by Böttcher, Schacht and Taraz [5]. It is natural to wonder to what extent the bounded maximum degree condition on $H$ can be relaxed. In particular, is it true that every $Q_{n}$-free graph on $2^{n}$ vertices has minimum degree at most $\left(\frac{1}{2}+o(1)\right) 2^{n}$ ? Note that $Q_{n}$ is a bipartite graph on $N=2^{n}$ vertices, which is $n$-regular with $n=\log N$, and has bandwidth $O(N / \sqrt{\log N})$. It appears that new techniques would have to be developed to handle this problem. The proof of the Bollobás-Komlós 
conjecture uses the regularity lemma and the blow-up lemma, giving quantitative estimates that are too weak to embed spanning subgraphs which are as dense as cubes. A positive solution would likely lead to a proof of the Burr-Erdős conjecture discussed above that cubes have linear Ramsey number.

Acknowledgements. We would like to thank the two anonymous referees for their valuable comments.

Note added in proof. Recently, the main conjecture studied in this paper, that $r\left(Q_{n}, K_{s}\right)=$ $(s-1)\left(2^{n}-1\right)+1$ for $s$ fixed and $n$ sufficiently large, was resolved by Fiz Pontiveros, Griffiths, Morris, Saxton and Skokan [17, 18]. Their proof builds upon the techniques developed in this paper.

\section{References}

[1] P. Allen, G. Brightwell and J. Skokan, Ramsey-goodness - and otherwise, Combinatorica 33 (2013), 125-160.

[2] N. Alon, P. Seymour and R. Thomas, A separator theorem for nonplanar graphs, J. Amer. Math. Soc. 3 (1990), 801-808.

[3] J. Beck, An upper bound for diagonal Ramsey numbers, Studia Sci. Math. Hungar. 18 (1983), 401-406.

[4] J. Böttcher, K. Pruessman, A. Taraz and A. Würfl, Bandwidth, expansion, treewidth, separators, and universality for bounded degree graphs, European J. Combin. 31 (2010), 1217-1227.

[5] J. Böttcher, M. Schacht and A. Taraz, Proof of the bandwidth conjecture of Bollobás and Komlós, Math. Ann. 343 (2009), 175-205.

[6] S. Brandt, Expanding graphs and Ramsey numbers, available at Freie Universität, Berlin preprint server, ftp://ftp.math.fu-berlin.de/pub/math/publ/pre/1996/pr-a-96-24.ps (1996).

[7] W. G. Brown, On graphs that do not contain a Thomsen graph, Canad. Math. Bull. 9 (1966), $281-285$.

[8] S. A. Burr, Ramsey numbers involving graphs with long suspended paths, J. London Math. Soc. (2) 24 (1981), 405-413.

[9] S. A. Burr, What can we hope to accomplish in generalized Ramsey theory?, Discrete Math. 67 (1987), 215-225.

[10] S. A. Burr and P. Erdős, On the magnitude of generalized Ramsey numbers for graphs, in: Infinite and Finite Sets I (Keszthely, 1973), Colloq. Math. Soc. Janos Bolyai, Vol. 10, 214-240, North-Holland, Amsterdam, 1975.

[11] S. A. Burr and P. Erdős, Generalizations of a Ramsey-theoretic result of Chvátal, J. Graph Theory 7 (1983), 39-51.

[12] S. A. Burr, P. Erdős, R. J. Faudree, C. C. Rousseau and R. H. Schelp, The Ramsey number for the pair complete bipartite graph-graph of limited degree, in: Graph theory with applications to algorithms and computer science (Kalamazoo, Mich., 1984), 163-174, Wiley, New York, 1985. 
[13] S. A. Burr, P. Erdős, R. J. Faudree, C. C. Rousseau and R. H. Schelp, Some complete bipartite graph-tree Ramsey numbers, Ann. Discrete Math. 41 (1989), 79-90.

[14] F. Chung and R. L. Graham, Erdős on Graphs. His Legacy of Unsolved Problems, A K Peters, Ltd., Wellesley, MA, 1998.

[15] V. Chvátal, Tree-complete graph Ramsey numbers, J. Graph Theory 1 (1977), 93.

[16] V. Chvátal and F. Harary, Generalized Ramsey theory for graphs, III. Small off-diagonal numbers, Pacific J. Math. 41 (1972), 335-345.

[17] G. Fiz Pontiveros, S. Griffiths, R. Morris, D. Saxton and J. Skokan, On the Ramsey number of the triangle and the cube, preprint.

[18] G. Fiz Pontiveros, S. Griffiths, R. Morris, D. Saxton and J. Skokan, The Ramsey number of the clique and the hypercube, preprint.

[19] J. Fox and J. Pach, A separator theorem for string graphs and its applications, Combin. Probab. Comput. 19 (2010), 371-390.

[20] J. Fox and B. Sudakov, Density theorems for bipartite graphs and related Ramsey-type results, Combinatorica 29 (2009), 153-196.

[21] J. Fox and B. Sudakov, Dependent random choice, Random Structures Algorithms 38 (2011), 68-99.

[22] R. L. Graham, V. Rödl and A. Ruciński, On graphs with linear Ramsey numbers, J. Graph Theory 35 (2000), 176-192.

[23] R. L. Graham, V. Rödl and A. Ruciński, On bipartite graphs with linear Ramsey numbers, Combinatorica 21 (2001), 199-209.

[24] A. Kostochka, The minimum Hadwiger number for graphs with a given mean degree of vertices, Metody Diskret. Analiz. 38 (1982), 37-58.

[25] A. Kostochka, Lower bound of the Hadwiger number of graphs by their average degree, Combinatorica 4 (1984), 307-316.

[26] A. Kostochka and V. Rödl, On graphs with small Ramsey numbers, J. Graph Theory 37 (2001), 198-204.

[27] R. J. Lipton and R. E. Tarjan, A separator theorem for planar graphs, SIAM J. Appl. Math. 36 (1979), 177-189.

[28] W. Mader, Homomorphiesätze für Graphen, Math. Ann. 178 (1968), 154-168.

[29] G. L. Miller, S.-H. Teng, W. Thurston and S. A. Vavasis, Separators for sphere-packings and nearest neighbor graphs, J. ACM 44 (1997), 1-29.

[30] V. Nikiforov and C. C. Rousseau, Ramsey goodness and beyond, Combinatorica 29 (2009), $227-262$. 
[31] N. Robertson and P. D. Seymour, Graph minors. XX. Wagner's conjecture, J. Combin. Theory Ser. B 92 (2004), 325-357.

[32] L. Shi, Cube Ramsey numbers are polynomial, Random Structures Algorithms 19 (2001), 99101.

[33] L. Shi, The tail is cut for Ramsey numbers of cubes, Discrete Math. 307 (2007), 290-292.

[34] R. Stanley, Enumerative Combinatorics, Volume 1, Cambridge Studies in Advanced Mathematics 49, Cambridge University Press, Cambridge, UK, 2002.

[35] A. Thomason, An extremal function for contractions of graphs, Math. Proc. Cam. Phil. Soc. 95 (1984), 261-265.

[36] A. Thomason, The extremal function for complete minors, J. Combin. Theory Ser. B 81 (2001), $318-338$. 